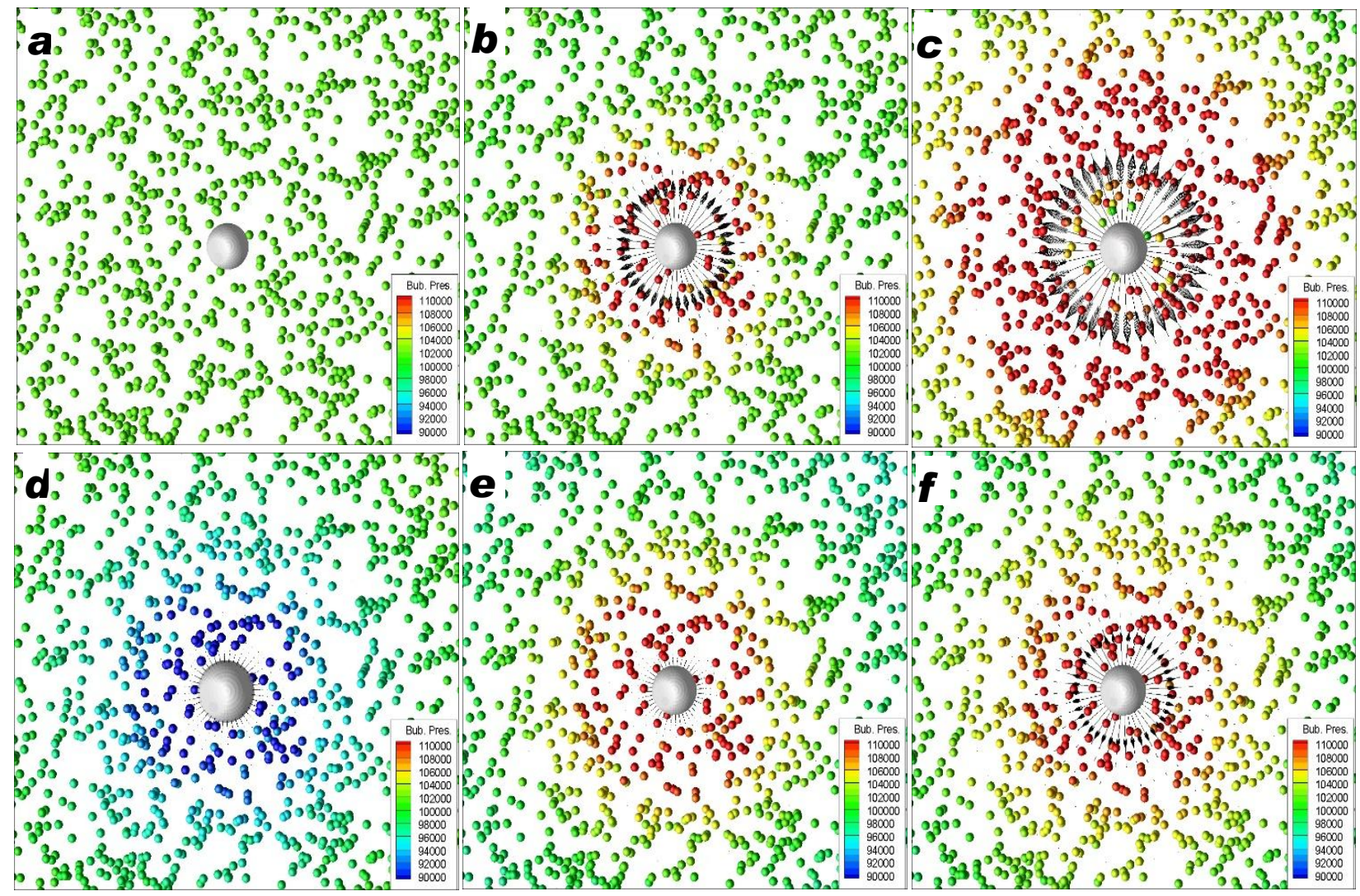




\title{
Spherical Bubble Dynamics in a Bubbly Medium using an Euler-Lagrange Model
}

\author{
Jingsen $\mathrm{Ma}^{\mathrm{a}}{ }_{*}$, Georges L. Chahine ${ }^{\mathrm{a}}$, and Chao-Tsung Hsiao ${ }^{\mathrm{a}}$ \\ ${ }^{a}$ DyNAFLOW, INC., 10621-J Iron Bridge Road, Jessup, MD, USA
}

\begin{abstract}
\end{abstract}
For applications involving large bubble volume changes such as in cavitating flows and in bubbly two-phase flows involving shock and pressure wave propagation, the dynamics, relative motion, deformation, and interaction of bubbles with the surrounding medium play crucial roles and require accurate modeling. We present in this paper a fundamental study of the dynamic oscillations of a 'primary' bubble in a bubbly mixture using a two-way coupled Euler-Lagrange model. It addresses a simplified spherical configuration while using the full three-dimensional code. A main objective of the study is to investigate how the dynamics of a 'primary' bubble is affected by the presence of a surrounding bubbly medium and how it differs from its behavior in a pure liquid. This helps elucidate the physics at play for this relatively simple configuration. The model simulates the mixture as a continuum and solves the corresponding Navier Stokes equations with grids moving with the interface of the primary bubble wall. The surrounding microbubbles are tracked in a Lagrangian fashion accounting for their volume evolution. The two-way coupling between the bubbly medium and the primary

* Corresponding author.

Emails: jingsen@dynaflow-inc.com; glchahine@dynaflow-inc.com; ctsung@dynaflow-inc.com Phone: 301-604-3688

Fax: 301-604-3689 
bubble dynamics is realized through the local density of the mixture obtained from the tracking of the microbubbles and the determination of their volumes and spatial distribution.

The simulations clearly indicate that the surrounding microbubbles absorb energy emitted from the primary bubble during its oscillations. This results in a reduction of the maximum radius and the period of oscillations of the primary bubble as compared to the dynamics in the pure liquid. Also, accounting for the dynamics of the field bubbles brings out the presence in the two-phase medium of a phase shift between density and pressure distributions. Such a shift is not captured by two-phase homogeneous medium models. These effects increase with increase in the mixture void fraction and in the initial bubble sizes in the mixture. The numerical observations are found to be in good qualitative agreements with previously published experimental data (Jayaprakash et al., 2011) investigating spark generated bubble dynamics in a bubbly medium.

Key Words: Bubble Dynamics, Bubbly Medium, Euler-Lagrange Model, Two-way Coupling, Phase Shift

\section{INTRODUCTION}

Bubbly flows are prevalent in chemical engineering processes and there are many engineering systems, where the dynamics of clouds of vapor or gas bubbles is of fundamental importance. For example, bubble columns reactors are used in a wide spectrum of chemical and energy applications (van Sint Annaland et al., 2005, Darmana et al., 2007, Law et al., 2008, Chen et al., 2009), with typical examples including Fischer-Tropsch synthesis (Guillen et al., 2011), methanol and dimethylether syntheses (Wu \& Gidaspow, 2000), coal liquefaction (Law et al., 2007, Law et al., 2011), or algae 
bioreaction (Singh \& Sharma, 2012) etc. Also, nucleate pool boiling is applied in different industrial processes such as distillation or thermal transport (Arndt, 1981, Blake \& Gibson, 1987). In addition, pipes containing air ventilated cavities are frequently encountered in engineering facilities including airlift bioreactors or U-tube fermenters (Xiang et al., 2011, Xiang et al., 2014). The use of cavitation for the intensification of bio-chemical processes is also very common such as in surface cleaning (Ohl et al., 2006), bacteria disinfection processes (Johnson et al., 1982, Kalumuck et al., 2003, Loraine et al., 2012), or biomedical therapy applications (Coleman et al., 1987, Zhong et al., 2001, Kennedy, 2005, Mitragotri, 2005, Chung \& Cho, 2009, Capretto et al., 2011, Yuan et al., 2011, Chahine \& Hsiao, 2012, Hsiao et al., 2013a, Madadi-Kandjani \& Xiong, 2014). Most of these above-mentioned problems involve large bubble volume changes due to boiling, cavitation and/or bubble breakup and coalescence. Particularly, in liquid phase oxidation processes such as injection of oxygen into liquid (Kalumuck \& Chahine, 2000) an explosion behavior occurs, followed by the emission of high-peak spherical pressure waves which propagates through the surrounding bubbly liquid. In these kinds of problems, shock and pressure wave propagation in bubbly media, the dynamics, relative motion, and interaction of bubbles with the surroundings play crucial roles on the process in question and require accurate modeling, which poses fundamental challenges.

Since the early works of Besant (1859) and Rayleigh (1917) a large body of literature has been devoted to the dynamics of spherical bubbles. Plesset (1949) presented a comprehensive model describing the time variations of the radius of a 
bubble containing compressible gas in an incompressible liquid when the liquid is subjected to a time dependent ambient pressure. The model conserves mass and momentum, includes surface tension and liquid viscous effects, but ignores gas diffusion and heat transfer between the bubble and the liquid. Since then, many researchers have extended this model to include other effects such as liquid compressibility (Herring, 1941, Gilmore, 1952, Keller \& Kolodner, 1956, Prosperetti \& Lezzi, 1986, Prosperetti, 1987), mass transfer (Prosperetti, 1982, Hsiao \& Chahine, 2012), heat transfer (Miksis \& Ting, 1984, Chahine \& Liu, 1985, Prosperetti et al., 1988, Commander \& Prosperetti, 1989, Prosperetti, 1991), electro-potential effects (Oh et al., 2001, Spelt \& Matar, 2006), magnetic effects (Yasui, 1999, Aliabadi \& Taklifi, 2012). Even though not directly applicable to the present study, several models have also been developed and applied to the study of non-spherical bubble dynamics (Chahine, 1982, Zhang et al., 1993, Hsiao \& Chahine, 2001, Choi et al., 2009, Wang \& Blake, 2010). The Rayleigh-Plesset equation (Rayleigh-Plesset equation) and its small compressibility equivalent models (Herring, 1941, Gilmore, 1952, Keller \& Kolodner, 1956), remain however, by far the most widely used models for a wide range of applications involving bubble dynamics such as hydrodynamic cavitating flows (Plesset, 1949, Ceccio \& Brennen, 1991, Brennen, 1995, Chahine, 2009, Brennen, 2011), acoustic cavitation applications (Keller \& Miksis, 1980, Hilgenfeldt et al., 1998, Moholkar et al., 1999, Prosperetti \& Hao, 1999, Calvisi et al., 2007), two-phase bubbly flows (Seo et al., 2010, Shams et al., 2011, Hsiao et al., 2013b), and underwater explosion bubbles (Herring, 1941, Keller \& Kolodner, 1956, Chahine \& Harris, 1997, Wardlaw \& Mair, 1998, Geers \& Hunter, 2002, Krieger \& Chahine, 2005, de Graaf et al., 2012). This is predominantly because of the success of these methods at recovering the key physics involved in each application and because of the simplicity of their implementation as compared to other models.

The incompressible Rayleigh-Plesset equation actually remains at the core of the solutions of most numerical simulation models of two-phase bubbly flows (Plesset \& Prosperetti, 1977). In most cases, as for the classical modeling of a two-phase flow in a 
nozzle, an equivalent medium approach is used with the equivalent density determined from the volume occupied by the bubbles and obtained with simplifying assumptions through local solution of the Rayleigh-Plesset equation (Van Wijngaarden, 1964, Brennen, 1995, Grandjean et al., 2012). In general, two-phase bubbly flows are modeled using one of the following approaches: an equivalent homogeneous continuum approach, an Eulerian two-fluid approach, or an Eulerian-Lagrangian approach wherein the bubbles are treated as discrete particles. While homogeneous models, which are useful for low void fraction and very small bubble oscillations conditions, ignore bubble dynamics and do not require Rayleigh-Plesset equation solutions, the other two approaches do require such modeling. Eulerian-Lagrangian approaches are more appropriate for higher void fractions (Spelt \& Biesheuvel, 1997, Balachandar \& Eaton, 2010, Raju et al., 2011, Shams et al., 2011). In a recent work by Raju et al. (2011) comparing a continuum homogeneous model (Gilmore, 1952), an Eulerian multicomponents model (Wardlaw \& Luton, 2000, Wardlaw \& Luton, 2003), and an Eulerian-Lagrangian model (Hsiao et al., 2003, Chahine, 2009, Hsiao et al., 2013b) it was found that high-frequency local fluctuations were only captured when an Eulerian viscous solver was coupled with a Lagrangian discrete bubble dynamics and when the microscale behavior of the field bubbles was well resolved. Continuum models, on the other hand, captured well the average low-frequency behavior. Proper discrete bubble modeling is thus essential to an accurate modeling of the problem (Sussman, 2003, Francois et al., 2006, Fuster \& Colonius, 2011, Lauer et al., 2012, Aanjaneya et al., 2013, Arienti \& Sussman, 2014).

In this paper, we utilize a generalized 3D two-way coupled Euler-Lagrange twophase flow model and apply it in a spherical configuration to study the dynamics of a primary bubble oscillating in a bubbly mixture. The 3D model has been developed to study bubbly and cavitating flows such as the effects of a propeller flow and dissolved gas diffusion into and out of the bubbles on the bubble size distribution in water (Hsiao \& Chahine, 2012), the development of tip vortex cavitation on propellers (Hsiao \& Chahine, 2008), bubbly flow in a bubble augmented propulsor (Wu et al., 2010), bubble 
entrainment in jet wake flows (Hsiao et al., 2013b), and has evolved from the study of highly deformable bubbles in vortices (Hsiao \& Chahine, 2001, Choi et al., 2009) and cavitating flow (Chahine \& Hsiao, 2000, Chahine, 2009).

The 3D two-phase mixture domain is gridded using an O-grid with a moving grid adapting to the moving primary bubble wall boundary. The equivalent continuum Navier-Stokes equations with local and time dependent local void fractions are solved in this domain. The shape of the surrounding bubbles is not the subject of particular attention in this study and these bubbles are treated in an approximated manner as singularities with the first mode being a source with its intensity satisfying the RayleighPlesset-Keller-Herring equations. Deformation of these bubbles is ignored, however volume change, motion and interactions, and their effects on the flow are well captured. The two-way coupling between the mixture flow and the dispersed bubbles is realized through the local mixture density, which is based on continual updating of the local volume and distribution of the dispersed bubbles. A Gaussian scheme is adopted to smooth the void fraction spatial distribution and derivatives. Using this model, the effects of the presence of the bubbles on the primary bubble dynamics and on the propagation of pressure and density waves in the medium are investigated. Various mixture conditions with void fractions ranging between $0 \%$ and $5 \%$ and field bubble sizes between one tenth and one fifth of the primary bubble size are studied. The configuration of the 3D problem is made practically spherically-symmetric by randomly distributing the field bubbles in a spherical computational domain while satisfying a statistically uniform void fraction distribution in space. This is for model verification purposes and to facilitate comparison with available analytical spherical bubble solutions. The model is however fully $3 \mathrm{D}$ and is not configuration limited. It is, for instance, also suitable to study non-spherical bubble dynamics (e.g., bubble collapse near a wall) in bubbly mixtures, which is illustrated briefly in the verification part of this paper and which will be the subject of a separate paper.

The paper is organized as follows. In the next section we describe the main features of the coupled Euler-Lagrange model, the void fraction computation method, and the 
void distribution smoothing scheme. We then present the bubble driven flow considered here and the results obtained using the simulation model, along with detailed discussions of field bubble effects on the primary bubble dynamics and on the wave propagation in the two-phase medium. Finally, we summarize the main findings from the study.

\section{EULER-LAGRANGE TWO-PHASE MODEL}

\subsection{Viscous Mixture Flow Solver}

The two-phase bubbly mixture with gas volume fraction, $\alpha$, is treated as a homogenous mixture with the following properties. The density, $\rho_{m}$, and kinematic viscosity, $\mu_{m}$, are defined respectively as:

$$
\begin{aligned}
& \rho_{m}=(1-\alpha) \rho_{l}+\alpha \rho_{g}, \\
& \mu_{m}=(1-\alpha) \mu_{l}+\alpha \mu_{g},
\end{aligned}
$$

where $\rho_{g}$, and $\mu_{g}$, are respectively the density and kinematic viscosity of the gas, and $\rho_{l}$, and $\mu_{l}$, are respectively the liquid density and kinematic viscosity.

Following the theoretical work on dilute bubbly flows of van Wijngaarden (1968, 1972), Commander \& Prosperetti (1989) and Brennen (2005), the unsteady continuity and momentum conservation equations for the liquid-gas homogeneous mixture are as follows:

$$
\begin{gathered}
\frac{\partial \rho_{m}}{\partial t}+\nabla \cdot\left(\rho_{m} \mathbf{u}\right)=0, \\
\rho_{m} \frac{D \mathbf{u}}{D t}=-\nabla p+\mu_{m} \nabla^{2} \mathbf{u}+\rho_{m} \mathbf{g},
\end{gathered}
$$

where $t$ is time, $\mathbf{u}$ is the mixture velocity, $p$ its pressure, and $\mathbf{g}$ is a body force such as the acceleration of gravity Note that Equations (2) and (3) are written for the mixture and not for the liquid. A different formulation is available in the literature, e.g. Ferrante \& Elghobashi (2004), Darmana et al. (2006), Shams et al. (2011), and applies the Navier 
Stokes equations to the liquid (not the mixture) and, as a result, requires an additional momentum exchange term. Here, a separate computation of the bubble motion and the drag force is conducted.

Equations (2) and (3) are solved using the flow solver 3 DYNAFS-VIS ${ }^{\odot}$, formerly called DFI-UnClE (Hsiao \& Chahine, 2001), which is based on the artificialcompressibility method (Chorin, 1967), where a derivative of the pressure in the pseudo time, $\tau$, is added to (2) as:

$$
\frac{1}{\beta} \frac{\partial p}{\partial \tau}+\frac{\partial \rho_{m}}{\partial t}+\nabla \cdot\left(\rho_{m} \mathbf{u}\right)=0
$$

where $\beta$ is an artificial compressibility factor. Equations (3) and (4) form a hyperbolic system of equations and are solved using a time marching scheme in the pseudo-time, $\tau$ . The time variations of the density is enforced as source terms in (3) and (4). To obtain a time-dependent solution, a Newton procedure where pseudo-time iterative stepping in $\tau$ is used at each physical time step, $t$, enables one to satisfy the continuity equation.

The numerical scheme uses a finite volume formulation. A first-order Euler implicit difference scheme is applied to the time derivatives. The spatial differencing of the convective terms uses a flux-difference splitting scheme based on Roe's method (Roe, 1981) and a van Leer's MUSCL method (Anderson et al., 1986, Van Leer et al., 1987) to obtain the first or third-order fluxes. A second-order central differencing is used for the viscous terms and the flux Jacobians are obtained numerically. The resulting system of algebraic equations is solved using a discretized Newton relaxation method in which symmetric block Gauss-Seidel sub-iterations are performed before the solution is updated at each Newton iteration. The 3 DYNAFS-VIS ${ }^{\odot}$ solver has been extensively validated and used to study a range of two-phase problems and its results have compared favorably with available experimental data (Chahine et al., 2008, Chahine, 2009, Hsiao et al., 2010). 


\subsection{Lagrangian Modeling of Dispersed Phase}

The bubbles dispersed in the field to form the two-phase medium are treated as point sources and dipoles to account for their volume change and translation relative to the liquid. Volume variations are obtained with a bubble dynamics equation using bubble surface averaged pressures and velocities, to account for flow non-uniformities, and an additional pressure term to account for bubble motion relative to the liquid (Hsiao et al., 2003, Chahine, 2009). The equivalent spherical bubble radius, $R(\mathrm{t})$, is obtained using a modified Rayleigh-Plesset-Keller-Herring equation (Plesset \& Prosperetti, 1977, Prosperetti \& Lezzi, 1986) which accounts for the compressibility of the surrounding bubbly medium and for flow field non-uniformities:

$$
\begin{aligned}
\left(1-\frac{\dot{R}}{c_{m}}\right) R \ddot{R}+ & \frac{3}{2}\left(1-\frac{\dot{R}}{3 c_{m}}\right) \dot{R}=\frac{\left(\mathbf{u}_{\text {enc }}-\mathbf{u}_{b}\right)^{2}}{4}+ \\
& \frac{1}{\rho_{m}}\left(1+\frac{\dot{R}}{c_{m}}+\frac{R}{c_{m}} \frac{d}{d t}\right)\left[p_{v}+p_{g_{0}}\left(\frac{R_{0}}{R}\right)^{3 k}-p_{\text {enc }}-\frac{2 \gamma}{R}-4 \mu_{m} \frac{\dot{R}}{R}\right]
\end{aligned}
$$

where $c_{m}$ is the local sound speed in the two-phase medium, $\gamma$ is the surface tension, and $p_{v}$ is the vapor pressure. $p_{g 0}$ is the initial gas pressure in the bubble corresponding to the initial bubble equivalent radius, $R_{0} . p_{\text {enc }}$ and $\mathbf{u}_{\text {enc }}$, the "encountered" pressure and velocity respectively, are the pressures and velocities in the two-phase medium averaged over the bubble surface. $\mathbf{u}_{b}$ is the bubble translation velocity. The introduction of $\mathbf{u}_{\text {enc }}$ and $p_{\text {enc }}$ in Equation (5) is to account for non-uniform pressures and velocities around the bubble and for slip velocity between the bubble and the host medium. Using $p_{\text {enc }}$ and $\mathbf{u}_{\text {enc }}$ instead of the conventionally used values at the bubble center, results in a major improvement over classical models (Hsiao et al., 2003, Chahine, 2009). We will concentrate here on purely inertial bubbles as for cavitation in liquid at ambient temperatures (Brennen, 1995) and ignore gas diffusion and heat transfer. Including these will be presented in other publications following our previous work as in (Chahine \& Liu, 1985). In "cold" liquids such as water at $20^{\circ} \mathrm{C}$, liquid vaporization occurs at a much smaller time scale than the bubble dynamics scale, vapor goes in and out of the 
bubble almost instantaneously, and the vapor inside the bubble remains constant and equal to the liquid vapor pressure at the considered temperature. On the other hand, the time scale of gas diffusion is very much larger than the bubble period and the amount of gas inside the bubble remains the same, which allows one to use the compression law with the constant $k$ in equation (5).

The bubble trajectory is obtained using the following equation of motion of the bubble in the liquid flow, which extends the (Johnson \& Hsieh, 1966) equation by adding a lift force on the bubble,

$$
\begin{aligned}
\frac{d \mathbf{u}_{b}}{d t} & =-\frac{3}{\rho_{l}} \nabla p-2 g+\frac{3}{4} \frac{C_{D}}{R}\left(\mathbf{u}_{e n c}-\mathbf{u}_{b}\right)\left|\mathbf{u}_{e n c}-\mathbf{u}_{b}\right| \\
& +\frac{3}{2 \pi} \frac{C_{L}}{R} \sqrt{\frac{\mu_{l}}{\rho_{l}}} \frac{\left(\mathbf{u}_{e n c}-\mathbf{u}_{b}\right) \times \boldsymbol{\omega}}{\sqrt{|\boldsymbol{\omega}|}}+\frac{3}{R}\left(\mathbf{u}_{e n c}-\mathbf{u}_{b}\right) \dot{R},
\end{aligned}
$$

where $\omega$ is the local vorticity, $C_{L}$ is the lift coefficient, and $C_{D}$ is the drag coefficient given by (Haberman \& Morton, 1953):

$$
\begin{gathered}
C_{D}=\frac{24}{R_{e b}}\left(1+0.197 R_{e b}{ }^{0.63}+2.6 \times 10^{-4} R_{e b}{ }^{1.38}\right), \\
R_{e b}=\frac{2 \rho_{l} R\left|\mathbf{u}_{e n c}-\mathbf{u}_{b}\right|}{\mu_{l}} .
\end{gathered}
$$

The last term in the right hand side of (6) is the Bjerknes force (Bjerknes, 1906) term due to coupling between bubble volume variations and bubble motion.

\subsection{Eulerian-Lagrangian Coupling}

The two-way coupling between the Eulerian continuum-based model and the Lagrangian discrete bubble model is realized through the following steps:

- The volume change of the individual bubbles in the flow field are controlled by the two-phase medium local properties and flow field gradients.

- The bubble motion in the flow field is controlled by the liquid flow field. 
- The local properties of the mixture (void fractions and local densities) are determined by the spatial distribution of the bubble and by their volumes.

- The mixture flow field with evolving mixture density distribution satisfies mass and momentum conservation.

The key to this coupling scheme is the deduction of a void fraction distribution from the instantaneous bubble sizes and locations. Initially, the void fraction was defined in each computational cell as the ratio of the total volume of bubbles in the cell by the cell volume. However, this was found not totally satisfactory because it often resulted in difficulty with differentiation due to the non-continuous character of $\alpha$ across cells (Wu et al., 2010, Raju et al., 2011)

Here, to smooth the discontinuities and improve stability, we apply a Gaussian gas volume distribution scheme to smoothly "spread" a bubble volume across neighboring cells as in (Kitagawa et al., 2001, Darmana et al., 2006, Shams et al., 2011, Capecelatro \& Desjardins, 2013). As illustrated in Figure 1, the void fraction computation scheme for a representative cell $i$ is obtained using the following expression:

$$
\alpha_{i}=\sum_{j=1}^{N_{i}} \frac{f_{i, j} V_{j}^{b}}{\sum_{k}^{N_{\text {cells }}} f_{k, j} V_{k}^{\text {cell }}},
$$

where $V_{j}^{b}$ and $V_{k}^{\text {cell }}$ are the volumes of the bubble $j$ and the cell $k$, respectively. $N_{i}$ is the total number of bubbles which are in the "influence range" of the cell $i$. $N_{\text {cells }}$ is the total number of cells "influenced" by bubble $j$, and $f_{i, j}$ is the weight factor of the contribution of bubble $j$ to cell $i$ based on distance. The weight factor is provided by the Gaussian distribution function:

$$
f_{i, j}=\frac{1}{(\sqrt{2 \pi} S)^{3}} e^{-\frac{\left|\mathbf{X}_{i j}\right|^{2}}{2 s^{2}}},
$$

where $\left|\mathbf{X}_{i, j}\right|$ is the distance between bubble $j$ and the center of cell $i$, and $S$ is the standard deviation. The derivation of the above expression (8), can be obtained as follows. The 
void contribution of a bubble $j$ to a neighboring cell $k$ can be obtained first by uniformly distributing the bubble volume to all the neighboring cells. This gives for the first approximation of the volume fraction due to bubble $j$ in cell $k$ :

$$
a_{k, 0}=\frac{V_{j}^{b}}{\sum_{k}^{N_{\text {cells }}} V_{k}^{\text {cell }}} .
$$

However, for a closer representation of the actual physics, the cells nearer to a given bubble should receive a larger contribution. To do so, we adjust (10) by using a weight function dependent on the distance between the bubble center and the cell center. This is achieved through the use of the Gaussian function in Equation (9). The modified approximation of the volume fraction due to bubble $j$ in cell $k$ then becomes:

$$
a_{k, 1}=\frac{V_{j}^{b}}{\sum_{k}^{N_{\text {cells }}} V_{k}^{\text {cell }}} f_{k, j} .
$$

However, $a_{k, 1}$ does satisfy conservation of the redistributed gas volume in the computational domain. To conserve the gas mass, we need to multiply $a_{k, 1}$ by a normalization factor, $W_{k}$, defined as the ratio of the volume of the bubble to the total gas volume in all cells, $N_{\text {cells }}$, i.e.,

$$
W_{k}=\frac{V_{j}^{b}}{\sum_{k}^{N_{\text {cells }}}\left(V_{k}^{\text {cell }} \alpha_{k}\right)}=\frac{V_{j}^{b}}{\sum_{k}^{N_{\text {cells }}}\left(V_{k}^{\text {cell }} \frac{V_{j}^{b}}{\sum_{k}^{N_{\text {cells }}} V_{k}^{\text {cell }}} f_{k, j}\right)}=\frac{\sum_{k}^{N_{\text {cells }}} V_{k}^{\text {cell }}}{\sum_{k}^{N_{\text {cells }}}\left(V_{k}^{\text {cell }} f_{k, j}\right)}
$$

This gives us the final expression of the volume fraction due to bubble $j$ in cell $k$ :

$$
a_{k}=\frac{V_{j}^{b}}{\sum_{k}^{N_{\text {cells }}}\left(V_{k}^{\text {cell }} f_{k, j}\right)} f_{k, j} \text {. }
$$

Finally, adding up the effects of all the $N_{i}$ bubbles influencing cell $i$, one obtains readily Equation (8) from Equation (13). 


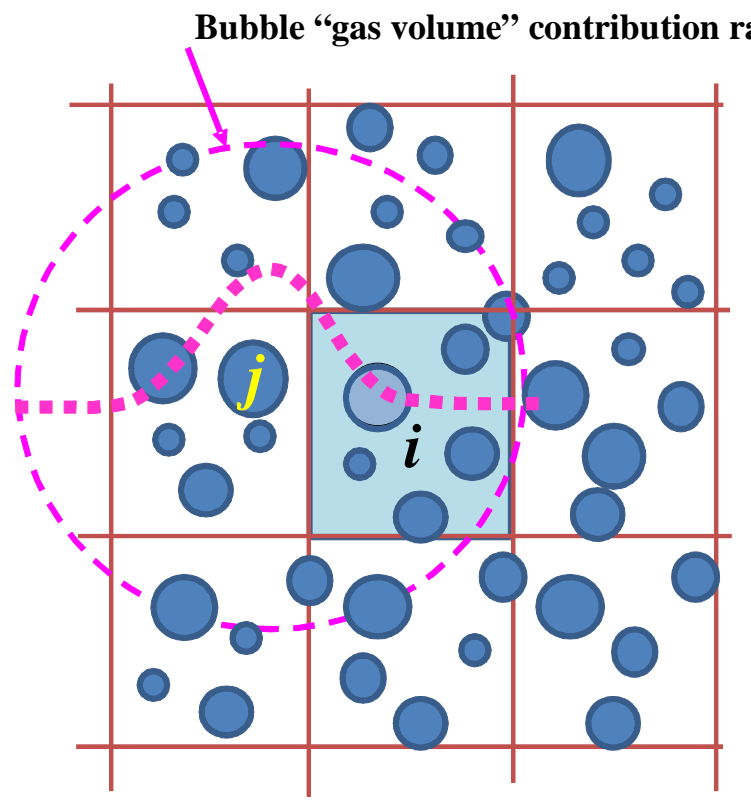

Figure 1. Illustration of the void fraction computation using the Gaussian distribution scheme.

\subsection{Primary Bubble Surface-Tracking Method}

In this study we consider conditions where the dynamics of the two-phase medium and of the dispersed bubbles are driven by the "primary" bubble behavior. In this case, it is essential to fully resolve the wall motion of this primary bubble and the high pressure and velocity gradients near it. Even though the physical problem considered here for verification purposes is spherical, a full 3D approach is taken. This allows to readily consider more complex configurations next. To do so, an explicit surfacetracking method using a moving grid technique is implemented and kinematic and dynamic free surface boundary conditions are imposed at the bubble-liquid interface. The Navier-Stokes equations are solved in the liquid domain containing the distributed field bubbles constrained by the free-surface boundary conditions at the primary bubble interface. 
The kinematic condition expresses that a fluid particle at the bubble-liquid interface remains on that surface. Specifically, if we define the equation of the free surface through the scalar function $F$,

$$
F\left(\xi_{i}, t\right)=\zeta-H(\xi, \eta, t)=0,
$$

where $\xi_{i}=(\xi, \eta, \zeta)$ are the curvilinear coordinates with $\zeta$ being in the direction normal to the bubble surface. The kinematic boundary condition can be written:

$$
\frac{D F}{D t}=\frac{\partial F}{\partial t}+U_{i} \frac{\partial F}{\partial \xi_{i}}=0,
$$

where $U_{i}=(U, V, W)$ are the contravariant velocity components.

Combining the equations (14) and (15) provides the general kinematic boundary condition in curvilinear coordinates as

$$
\frac{\partial H}{\partial t}=\left(W-\frac{\partial \zeta}{\partial t}\right)-U \frac{\partial H}{\partial \xi}-V \frac{\partial H}{\partial \eta} .
$$

The free surface dynamic boundary condition imposed at the bubble surface include balance of the normal stresses and vanishing shear stresses since we neglect the shear due to the gas inside the bubble. By requiring the grid to be normal to the interface boundary and assuming that the tangential derivatives of the normal velocity to the interface, $\partial W / \partial \xi$ and $\partial W / \partial \eta$, are negligible relative to the other derivatives, we use the Batchelor (1967) and Hodges et al. (1996) simplification to obtain:

$$
\begin{gathered}
\left.\frac{\partial U}{\partial \zeta}\right|_{\zeta=0}=0, \\
\left.\frac{\partial V}{\partial \zeta}\right|_{\zeta=0}=0, \\
p=p_{g}+p_{v}+\rho_{m} g z-\gamma \circlearrowright-\left.\mu_{m} \frac{\partial W}{\partial \zeta}\right|_{\zeta=0} .
\end{gathered}
$$

where $p$ is the liquid pressure at the bubble surface, $p_{g}$ and $p_{v}$ are the gas and vapor pressure inside the bubble respectively, and $\mathcal{C}$ is the interface curvature. 
A combination of algebraic and elliptic grid generation techniques (Hsiao, 1996, Hsiao \& Pauley, 1998) is used to update the position of the moving grid as the primary bubble changes volume and the interface points move away from or towards the origin. The algebraic technique allows clustering and boundary-orthogonal grids near the bubble surface. This is important for resolving the flow field near the bubble wall and

for properly applying the free surface boundary conditions. In addition, the elliptic grid generation techniques smooth out any roughness resultant from the algebraic technique and improve numerical stability.

\section{SIMULATION SETUP}

A primary bubble of radius $R_{0}$ is placed in a bubbly mixture composed of randomly distributed bubbles in an open 3D domain to create an initial quasi uniform void fraction distribution in space (Figure 2). In this paper, we will consider cases with field bubbles of the same size. This is not a constraint in the actual software and is used here only to simplify the presentation of the results. This setup resembles previously studied spherical bubble clouds (Mørch, 1981, Chahine, 1983, Chahine \& Liu, 1985, Chahine \& Duraiswami, 1992, Brennen, 1995, Ma et al, 2015). However, one notable difference is that here the central bubble (primary bubble) acting as the motion/pressure source is the initial driver of the motion. It then responds to the behavior of the surrounding bubbles. This also corresponds to our experimental tests of a spark generated bubble in a bubbly medium (Jayaprakash et al., 2011). In this study, the other bubbles' effects are included through their locations and volume changes while shape effects are ignored. These effects can be included, using the same code, at a much larger computational cost (Hsiao \& Chahine, 2001, Chahine, 2009, Choi et al., 2009, Hsiao et al., 2010). 


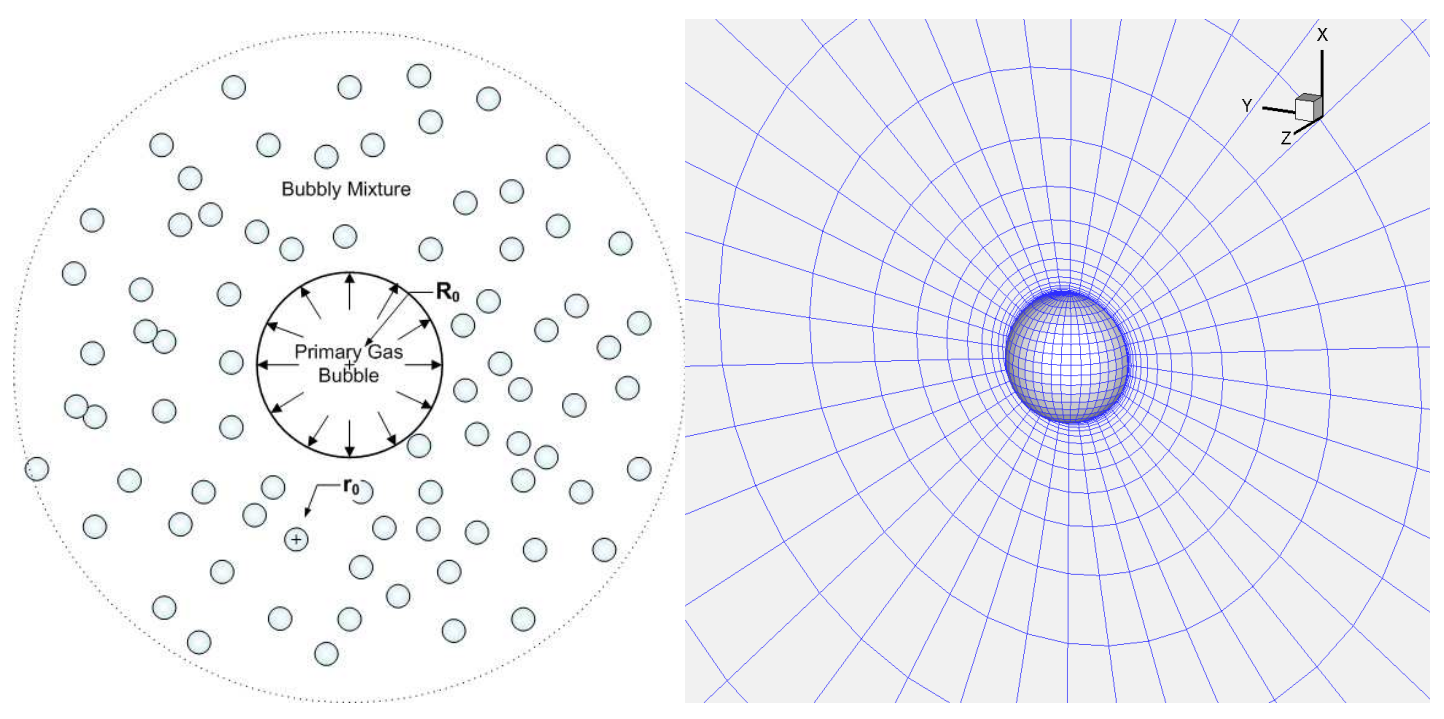

Figure 2: Schematic showing the problem configuration of an oscillating primary bubble in a bubbly mixture (Left), and grid used for the solution of the Eulerian two-phase flow mixture (Right). Note that the bubble interface and the grids are recomputed at each time step.

To study the dynamics of a spherical bubble cloud configuration we zero the gravity term in the input to prevent the gravity body force from altering the spherical symmetry. An O-O type single block grid is used as illustrated in Figure 2. The selected liquid interface discretization of the primary bubble has $41 \times 21$ nodes and the fluid domain has 25 nodes distributed in the radial direction between $r=R_{0}$ and the radius of the computational domain selected to be $100 R_{0}$. The grid in the radial direction is clustered near the bubble surface. This grid has been shown to be fine enough to provide gridconverged solutions for problems similar to that studied here (Raju et al., 2011). In the far field a discretized boundary is used at $r=\mathrm{A} R_{0}$ and a zero-gradient condition is imposed. A value of $A=100$ was found acceptable as illustrated in Figure 3. This considers the problem of a primary bubble of initial radius $R_{0}=5 \mathrm{~mm}$ and initial gas pressure $P_{g 0}=2 \mathrm{~atm}$ released in a bubbly mixture initially in equilibrium with the liquid ambient pressure $P_{a m b}=1 \mathrm{~atm}$ of water. The initial conditions of pressure and velocity in the medium outside the primary bubble correspond to quiescent conditions, i.e., $P=P_{a m b}$, $u=0$. Figure 3 shows that changing the limit of the domain size from $100 R_{0}$ to $300 R_{0}$ 
does not affect the solution in any significant fashion. The value $100 R_{0}$ is therefore used for the following computations.

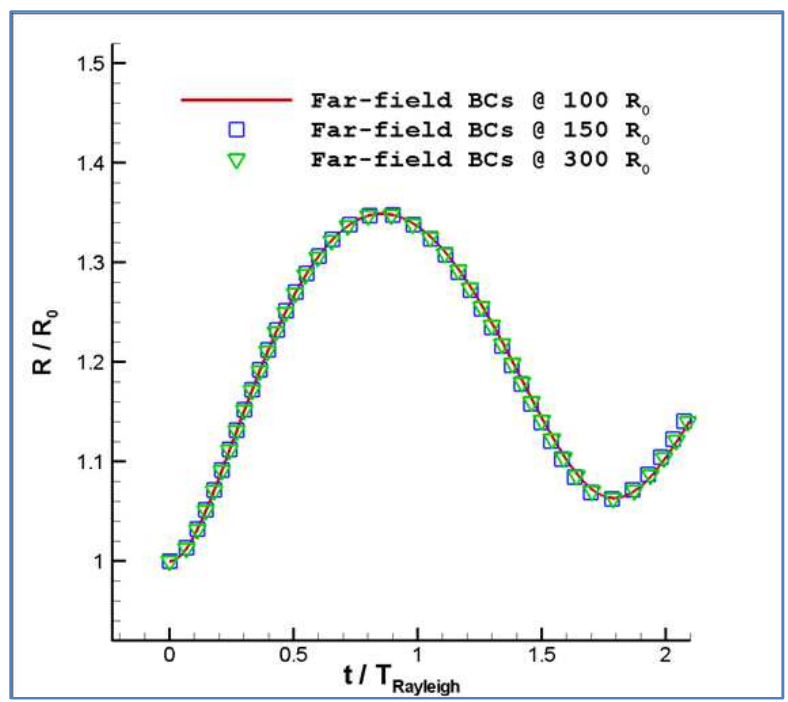

Figure 3: Effect of the domain size on the primary bubble radius versus time for the case of

$$
R_{0}=5 \mathrm{~mm}, P_{g 0}=2 \mathrm{~atm}, \alpha_{0}=1 \% \text { and } a_{0}=1 \mathrm{~mm} .
$$

We consider below the behavior of this bubbly medium and of the primary bubble for initial void fractions, $\alpha_{0}$, ranging from 0 to $5 \%$ and for initial radii, $a_{0}$, and for three field bubbles initial uniform radii: $0.5,0.75$ and $1 \mathrm{~mm}$. The bubbles are filled of air with density is $1.2 \mathrm{~kg} / \mathrm{m}^{3}$ and the file bubbles are considered in equilibrium at the initiation of the computations and have an initial gas pressure given by $P_{g 0}=P_{a m b}+2 \gamma / a_{0}-p_{v}$, where $P_{a m b}$ is the liquid ambient pressure, $\gamma$ is the surface tension parameter with the value $\gamma=0.0728 \mathrm{~N} / \mathrm{m}$ used in the numerical computations, and $p_{v}$ is the vapor pressure with the value $p_{v}=2,300 \mathrm{~Pa}$. The initial bubble wall and translation velocities are all set to zero.

For all the simulations presented in this paper, the artificial compressibility factor $\beta$ in Equation (4) is chosen to be 200, which was found to be large enough to achieve parameter independency, i.e., further increase of $\beta$ does not affect the simulation results. This is illustrated in Figure $4 \mathrm{a}$, which shows the effect of $\beta$ on the primary bubble 
equivalent radius. The results are seen to have already practically converged for $\beta=100$ and to not change much for $\beta$ larger than 100 . The solutions can also obviously be affected by the selected size of the time steps - here not selected to be adaptive for better understanding of the effect of the numeerical procedures. The effect of the time step size, $\delta t$, is shown in Figure $4 \mathrm{~b}$. The solutions overlap for time steps smaller than $3 \mu \mathrm{s}$, which correspond to $\delta t \simeq T_{\text {Rayleigh }} / 350$, where by analogy to the Rayleigh collapse time of an bubble of initial size $R_{0}$, we define a $T_{\text {Rayleigh }}$, which corresponds to the full period of a gaseous bubble of initial size $R_{0}$, in a liquid of density $\rho_{l}$, and an initial positive pressure difference between the initial gas pressure in the bubble, $P_{g 0}$, and the ambient pressure, $P_{a m b}$,

$$
T_{\text {Rayleigh }}=2 R_{0} \sqrt{\frac{\rho_{l}}{P_{g 0}-P_{a m b}}} .
$$

Based on the above, a value of $\delta t=1.5 \mu$ s is selected for the verification study illustrated below in Figure 4 and all the simulations presented hereafter.
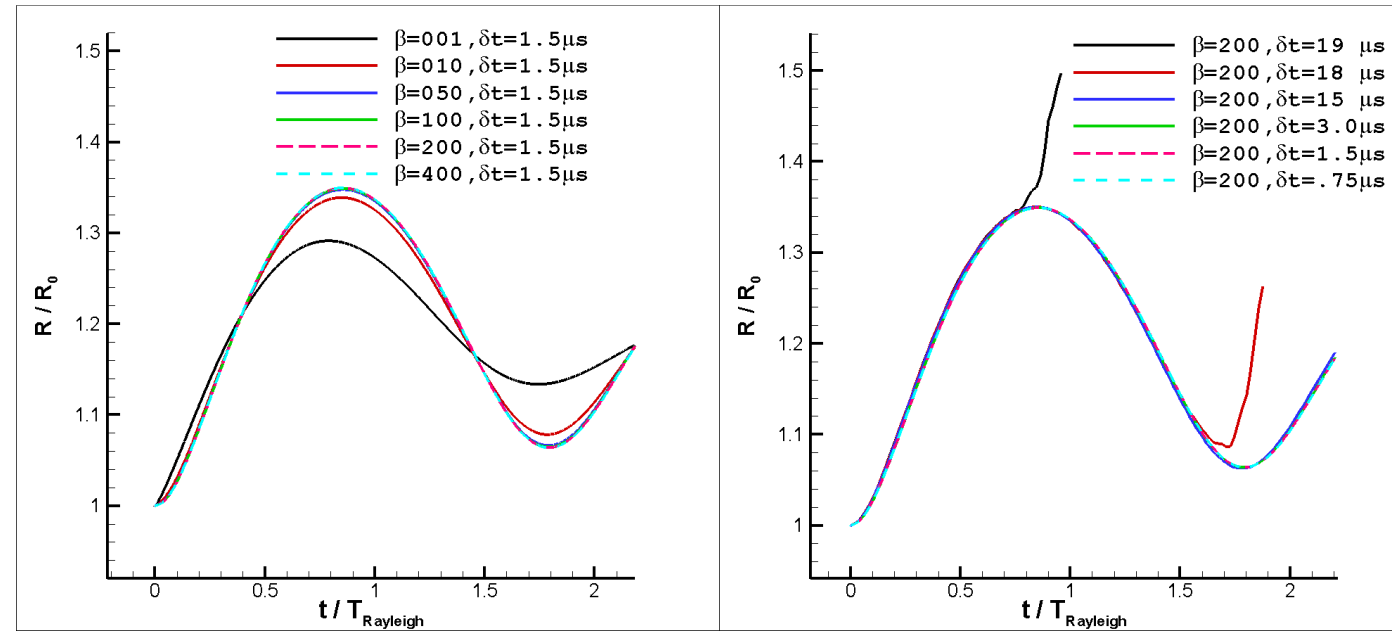

Figure 4: Effect of the numerical parameters in the viscous problem solution on the primary bubble radius versus time. (Left) Effect of the artificial compressibility parameter $\beta$ in Equation (4). (Right) Effect of the time step size on convergence. $R_{0}=5 \mathrm{~mm}, P_{g 0}=2 \mathrm{~atm}$. Bubbly mixture conditions: $\alpha_{0}=1 \%$ and $a_{0}=1 \mathrm{~mm}$ 


\subsection{Shape of the primary bubble}

Since the primary bubble and surrounding bubbly medium were selected to simulate a spherical bubble behavior while the code used is fully three-dimensional, it is useful to examine any deviation with time of the resulting primary bubble shape from a sphere. The shape of the primary bubble at three instants during the dynamics is shown in Figure 5 for the base case considered here $\left(R_{0}=5 \mathrm{~mm}, P g_{0}=2 \mathrm{~atm}, P_{a m b}=1 \mathrm{~atm}\right.$, initial void fraction, $\alpha_{0}=1.0 \%$, and initial "field" bubble radius $a_{0}=1 \mathrm{~mm}$ ). The figure indicates a visually very smooth spherical bubble shape at the three selected instances during the bubble period: $t=0, t=0.5 T_{0}$, and $t=T_{0}$.
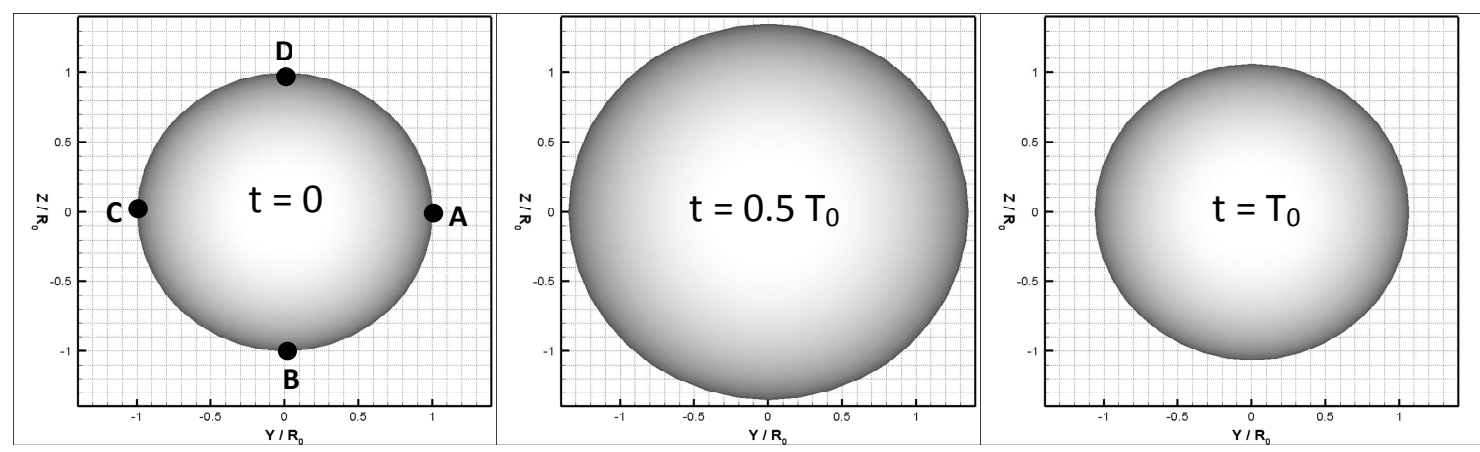

Figure 5: Primary bubble shape at different times during the first period of oscillations: (a) $t=0$, (b) $t=0.5 T$ and (c) $t=1.0 T$. $\alpha_{0}=1.0 \%, a_{0}=1 \mathrm{~mm}, R_{0}=5 \mathrm{~mm}, P g_{0}=2 \mathrm{~atm}, P_{a m b}=1 \mathrm{~atm}$.

This is further demonstrated quantitatively in Figure $6 \mathrm{a}$ which compares the temporal variations of the coordinates of four selected points on the bubble surface (at $X=Y=0$ and $X=Z=0$ ). The four points appear to very closely follow the same dynamics. Figure $6 \mathrm{~b}$ shows the deviation of the radial distances of the four points from the equivalent bubble radius, their averaged value versus time. We can see that these deviations do not exceed $0.23 \%$, which clearly indicates that the bubble remains very close to a sphere. These minor deviations are due to the non-perfectly spherically symmetric configuration generated by the random distribution of field bubbles. This facilitates the comparative analysis of the simulation with analytical equations such as 
the Gilmore equation which only deals with spherical bubble dynamics. Thus, any differences found between the two methods should be attributed to the inclusion of the individual field bubble dynamics in the modeling presented here, which plays no role in homogenous medium analytical methods.

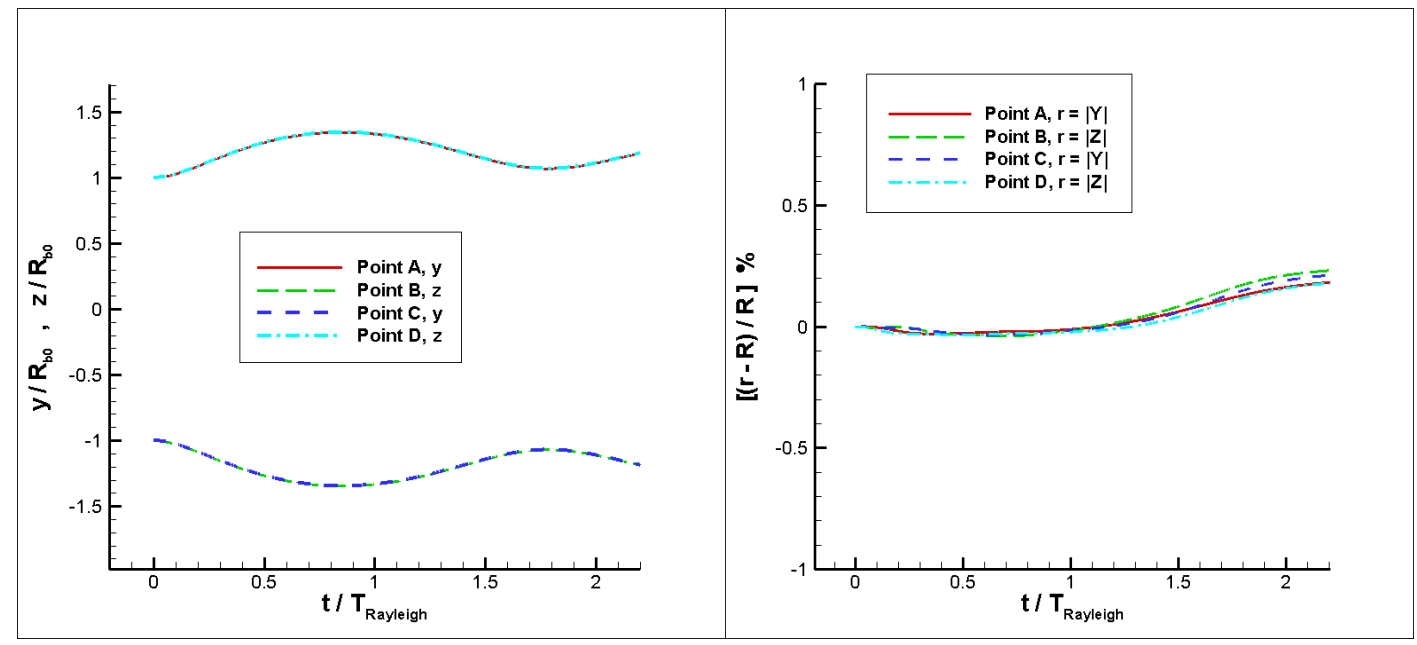

Figure $6:$ (a) Temporal variation of the coordinates of the four representative points on the bubble surface, points A, B, C, D in Figure 5 with $Y=0$ and $Z=0$. (b) Deviation of the radial distances of the four points from the equivalent bubble radius, $\mathrm{R}$, versus time.

\section{VERIFICATION CASES}

In order to verify the present Euler-Lagrange two-phase model, we first compare the time variations of the primary bubble radius in pure water and in the bubbly medium with the analytical solution of the bubble dynamics equations in a homogenous medium developed by Gilmore (Gilmore, 1952). To apply the Gilmore equations for a two phase medium, the dependencies of the density and the sound speed on the void fraction (Brennen, 1995) are incorporated in the solution of the equations (Raju et al., 2011).

As displayed in Figure 7, the results obtained by two methods match with each other very well for both pure water and the bubbly medium, here with $\alpha_{0}=1 \%$ and field bubble sizes $a_{0}=1 \mathrm{~mm}$. Some phasing differences appear between the two methods in presence of the field bubbles, but these differences are not too large for this void fraction and bubble size conditions. We will see later that the results between the 
two methods will deviate when larger non-linear effects due to the field bubbles, which are captured by the present model and ignored by a homogeneous approach, come into play, e.g. for higher void fractions.

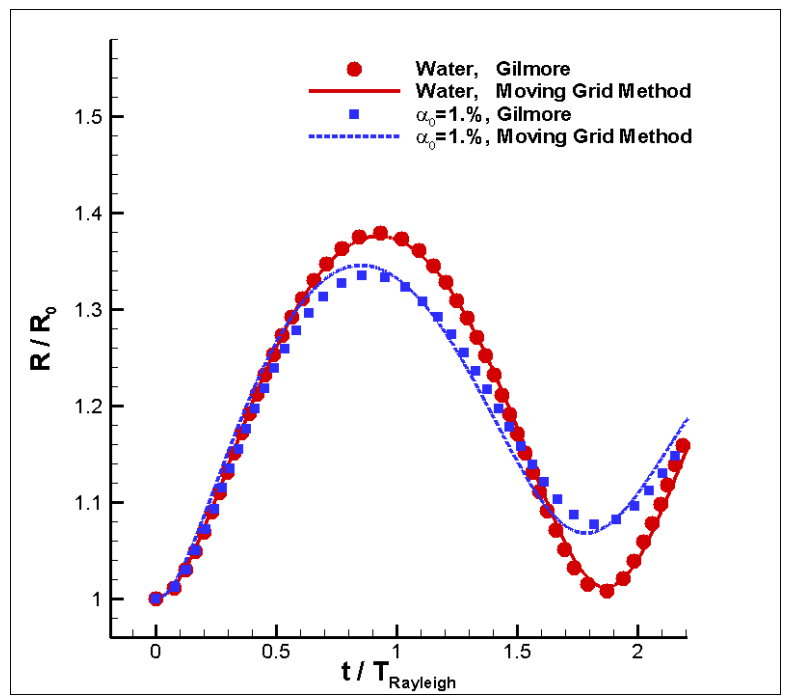

Figure 7: Comparison of the primary bubble radius versus time results of the present method and the solution of Gilmore equation. $R_{0}=5 \mathrm{~mm}, P_{g 0}=2 \mathrm{~atm}, P_{a m b}=1 \mathrm{~atm}$. Bubbly mixture conditions: $\alpha_{0}=1 \%$ and $a_{0}=1 \mathrm{~mm}$
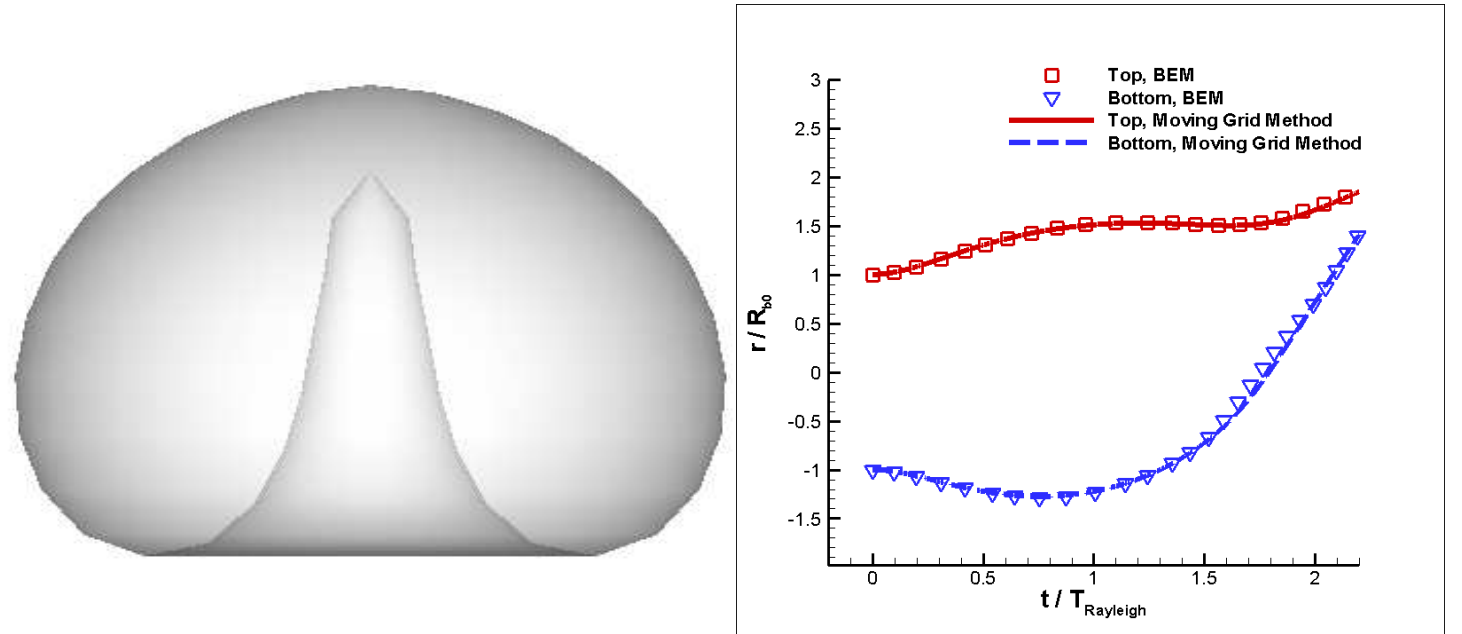

Figure 8: (Left) Shape of bubble with a reentrant jet under a Froude number $F_{r}=20$ predicted by 3DYNAFS-VIS ${ }^{\odot}$. (Right) Comparison between the boundary element method, 3DYNAFS-BEM ${ }^{\Theta}$, and the viscous code, $3 \mathrm{DYNAFS} \mathrm{VIS}{ }^{\odot}$, computations for the motion of the two bubble poles. $F_{r}=1.5, R_{0}=5 \mathrm{~mm}, P_{g 0}=2 \mathrm{~atm}, a_{0}=1 \mathrm{~mm}, P_{a m b}=1 \mathrm{~atm}$. 
In order to demonstrate that the present method applies to general 3D problems, and is not limited to spherical problems (subject of this paper) we consider its verification for a problem involving a collapsing bubble with a strong reentrant jet formation. To do so, we consider the same primary bubble initial conditions as in the previous case above in the pure liquid and impose on the bubble a body force, $g$, as in (3), selected to be strong enough to produce a significant jet. The relative effect of the body force is illustrated through the Froude number, $F_{r}$, defined as:

$$
F_{r}=\frac{P_{g 0}}{\rho g R_{0}} .
$$

In the example presented here, $F_{r}$ is set to be 20 . As illustrated in the $3 \mathrm{D}$ bubble shape in Figure 8(left), a significant and well developed jet can be seen at the end of the computation when the bubble is about to become multi-connected. To check the results, the solution of the 3DYNAFS-Vis ${ }^{\odot}$ Navier-Stokes computation with the moving grid method presented here is compared to the solution of the three-dimensional potential flow solver, 3DYNAFS-BEM ${ }^{\odot}$, which is based on the Boundary Element Method. This method is known to be very accurate for reentrant jet problems and has been fully validated with tests of cavitation bubbles, laser and spark-generated bubbles, and underwater explosion bubble dynamics (Zhang et al., 1993, Chahine et al., 1996, Chahine \& Kalumuck, 1998). As illustrated in the quantitative comparison in Figure 8(right), the present method very well reproduces the upward reentrant jet dynamics. The figure shows the time evolution of the location of the bubble top and bottom points (on the axis of symmetry, which indicates that the direction of application of the body force is vertical). Overall, during the full bubble period (growth and collapse with reentrant jet development) the two methods show very good agreement and do not deviate by more than a couple of percent. 


\section{PRIMARY BUBBLE DYNAMICS AND INTERACTION WITH MEDIUM}

\subsection{Dynamics of primary bubble and surrounding bubbles}

In order to illustrate the physics at play during the dynamics of a primary bubble in a responding two-phase bubbly medium, Figure 9 displays a color plot at different time instances corresponding to the first primary bubble oscillation period, $T_{0}$. Here we used $T_{0}$ to mark the time instants to highlight the temporal evolution within a period of primary bubble oscillation. The plot shows relative bubbles sizes and internal pressures, which also indicate liquid pressures. This corresponds to the same case shown earlier: $\alpha_{0}=1 \%, a_{0}=1 \mathrm{~mm}, R_{0}=5 \mathrm{~mm}, P_{g 0}=2 \mathrm{~atm}$, and $P_{a m b}=1 \mathrm{~atm}$. Overall, the randomly distributed field bubbles respond in a quasi-spherical mode as the pressure wave due to the primary bubble initial overpressure propagates radially out from the bubble. The corresponding velocity field moves the fluid particles outward during the primary bubble growth and then inward during the collapse.

As the pressure wave propagates out, the field bubbles, in particular those near the primary bubble interface, first see a pressure rise due to the higher initial pressure inside the primary bubble and respond by executing forced oscillations composed of their own natural frequency and that imposed by the primary bubble pressure field.

In the following we discuss some details of the dynamics of both the primary and surrounding bubbles at the different stages corresponding to snapshots a through $\mathbf{f}$ in Figure 9. In addition, to help a better understanding of the physics, we will refer to Figure 10, which shows the radial distributions of the pressures in the two-phase medium at different instants and the temporal histories of the medium velocity at a point not too far from the primary bubble wall, at $r=2.8 R_{0}$. 


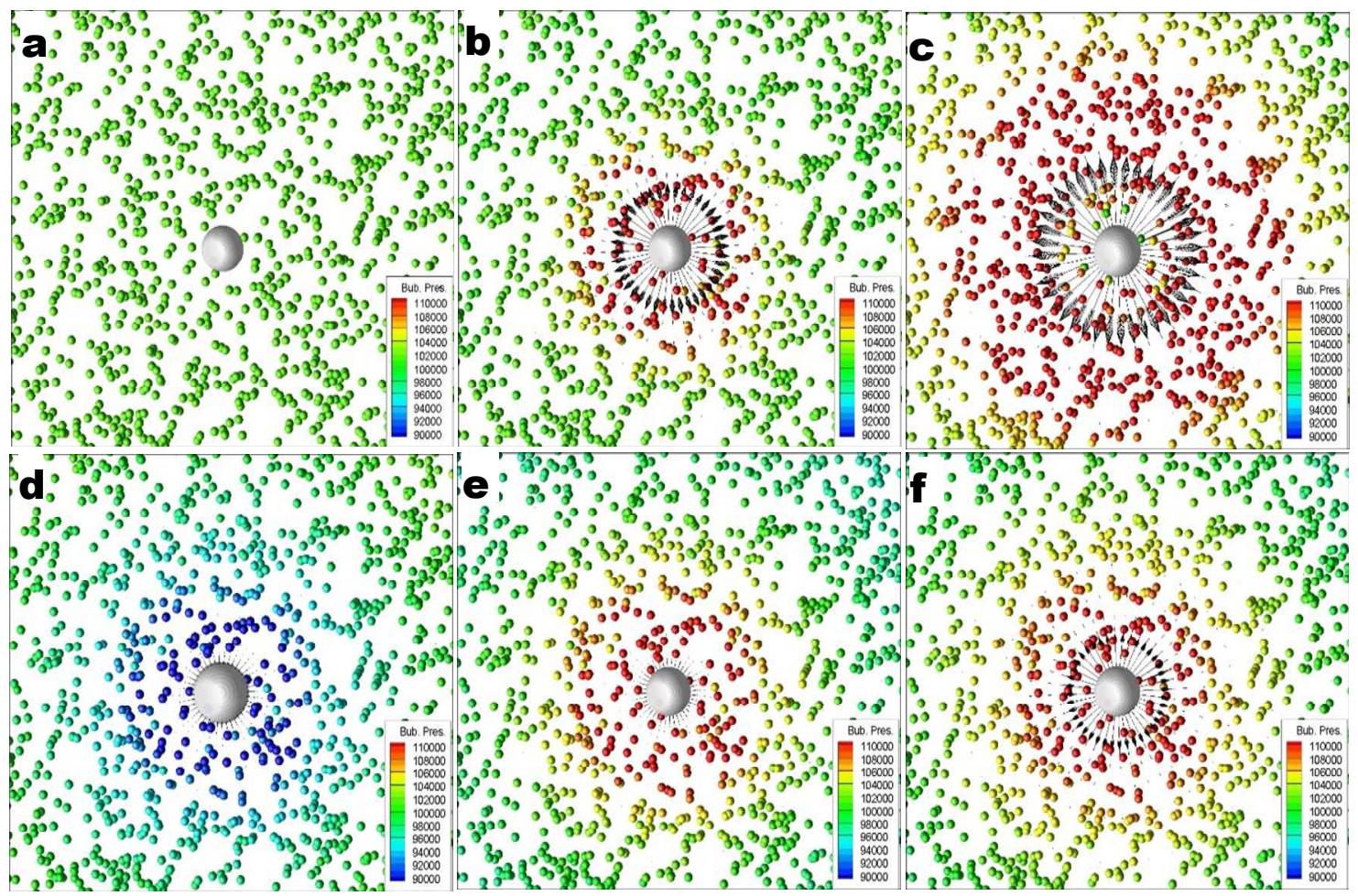

Figure 9: Time sequence of the bubbles' motion, volume change, and internal pressure variations during one primary bubble oscillating period, $T_{0}$ : (a) $t=0$, (b) $t=0.02 T_{0}$, (c) $t=0.15$ $T_{0}$, (d) $t=0.5 T_{0}$, (e) $t=1.0 T_{0}$, (f) $t=1.1 T_{0}$. The colors represent the bubble pressures and the arrows indicate the liquid velocities in the vicinity of the primary bubble. $\alpha_{0}=1.0 \%, a_{0}=1 \mathrm{~mm}, R_{0}=5 \mathrm{~mm}, P_{g 0}=2 \mathrm{~atm}, P_{a m b}=1 \mathrm{~atm}$.

Figure 9a corresponds to $t=0$, the initial state before the primary bubble wall is allowed to move. The initial internal gas pressure is $P_{g 0}=2 \mathrm{~atm}$. All the other field bubbles are at equilibrium with the ambient pressure, $P_{a m b}=1 \mathrm{~atm}$, accounting for surface tension, i.e. their initial gas pressure is given by $p_{g 0}=P_{a m b}+2 \gamma / a_{0}-p_{v}$. Following the release of the primary bubble wall, the liquid flows radially out (see the arrows in Figure $9 \mathrm{~b}$ and the velocity at time $\mathbf{b}$ in Figure 10b due to the suddenly imposed higher pressure at the boundary of the fluid domain. As a result, a radial pressure gradient develops in the medium as illustrated Figure 10a in a showing the radial pressure profile at $t=0.02 T_{0}$ near the primary bubble wall. As a result, the field bubbles close to the primary bubble wall experience compression and the gas pressure 
inside these bubbles increase as seen in Figure 9b. As the primary bubble continues its growth, the fluid velocities in the two-phase medium, e.g. at $r=2.8 R_{0}$, (Figure 10b), increase until the radially outward pressure gradient drops to zero and the velocity reaches a maximum value (Figure 9c). The timing of this maximum velocity depends on the radial location. For the region near the primary bubble wall, e.g., at $r=2.8 R_{0}$ this happen at about $t=0.15 T_{0}$ as seen in Figure 10. Then, due to inertia, the outward motion of the liquid and of the bubbles in the field continues but with decreasing liquid speeds, until the primary bubble reaches its maximum size at $t=0.5 T_{0}$ (Figure 9d). This outward motion is clearly illustrated between times $\mathbf{c}$ and $\mathbf{d}$ on the radial velocity curve in Figure 10b.

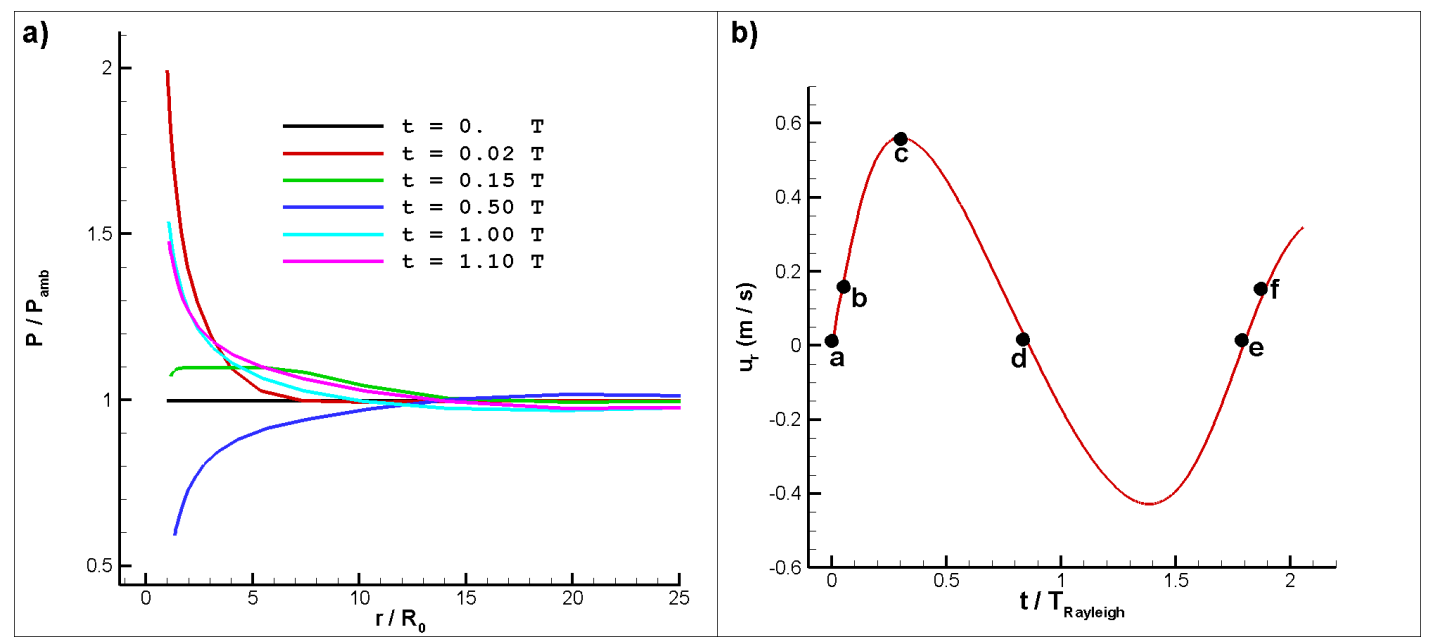

Figure 10: (a) Radial distribution of the pressure at different times during the first cycle of oscillations of the primary bubble. (b) Temporal variation of the radial component of the fluid velocity, $u_{r}$, monitored at $r=2.8 R_{0}$ for the case $\alpha_{0}=1.0 \%, R_{0}=5 \mathrm{~mm}, P_{g 0}=2 \mathrm{~atm}, a_{0}=1 \mathrm{~mm}$, $P_{a m b}=1 \mathrm{~atm}$. (The dashed line in the left graph corresponds to $r=2.8 R_{0}$ and the indices, a through $\mathbf{e}$, in the right graph correspond to the same time instances a through $\mathbf{e}$, in Figure 9.)

At $t=0.5 T_{0}$, the primary bubble starts to collapse after its pressure reaches a minimum. An opposite (negative) pressure gradient in the two-phase medium pointing towards the primary bubble center develops (see the radial pressure distribution curve corresponding to $t=0.5 T_{0}$ in the Figure 10a) and both the liquid and the field bubbles 
start to move inward until the primary bubble reaches a minimum size and its internal pressure reaches a maximum at $t=1.0 T_{0}$ (Figure 9e). During this collapse process the primary bubble recovers back most of its potential energy (but for viscous, and acoustic losses) and another cycle starts following bubble rebound (Figure 9f). All these observations are in line with classical bubble dynamics and with our previously published experiments of spark generated bubbles in bubbly media (Jayaprakash et al., 2011), and preliminary Euler-Lagrange coupled modeling work (Raju et al., 2011).

\subsection{Dynamics of the field bubbles}

In this section we turn our attention to the bubbles in the two-phase medium. Figure 11 illustrates the behavior by showing the temporal variations of the radius and position of four selected bubbles in the medium located initially at the following radial locations: $r$ $=1.8 R_{0}, r=2.8 R_{0}, r=3.8 R_{0}$, and $r=11 R_{0}$, for the same base condition as in Figure $9\left(\alpha_{0}\right.$ $\left.=1.0 \%, \mathrm{a}_{0}=1 \mathrm{~mm}\right)$. Each of the figures shows together the bubble center motion and the bubble radius versus time. These clearly highlight the field bubble response to the primary bubble dynamics. The amplitude of the responses increases when the distance to the primary bubble center is reduced. The field bubbles in the two-phase medium appear to exhibit their own resonance frequency, here about five times that of the primary bubble (since their initial radius is five times smaller than that of the primary bubble). Since the field bubbles are under forced oscillation, they oscillate at a frequency composed of the forcing frequency (i.e. that of the primary bubble) and their own natural frequency (i.e. here about the fifth harmonic of the primary bubble). On the other hand, the variations of the bubble locations and their radial distance to the primary bubble, appear to be dominated by the pressure/velocity flow field of the primary bubble dynamics. Figure 11 provides a comparison of the results for the bubbles at various initial distances from the center. As the distance from the primary bubble increases and the pressure variations are reduced, the amplitudes of the bubble radial oscillations at its own natural frequency are reduced and the bubble is seen to mainly respond almost in 
sync with the primary bubble pressure field. This effect is stronger on the bubble motion and, as a result, a clear shift between the phasing of the bubble motion and the bubble volume change can be seen increasing as the distance from the center increases.

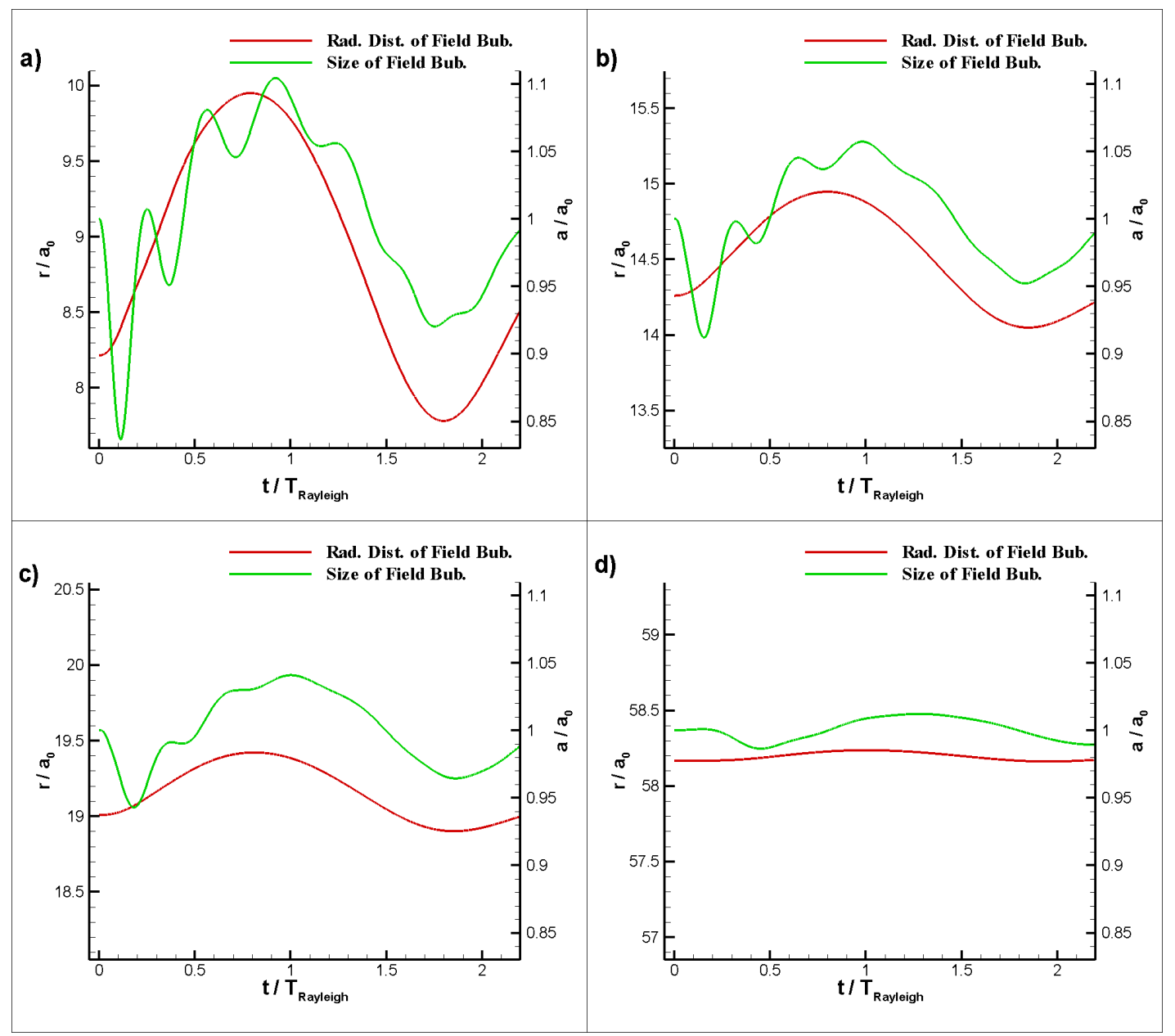

Figure 11: Radius and radial location versus time of field bubbles initially located at (a) $r=$ $1.6 R_{0}$, (b) $r=2.8 R_{0}, \quad(\mathrm{c}) \quad r=3.8 R_{0}$ and (d) $r=11 R_{0} . \alpha_{0}=1.0 \% . \quad R_{0}=5 \mathrm{~mm}$, $P_{g 0}=2 \mathrm{~atm}, a_{0}=1 \mathrm{~mm}, P_{\text {amb }}=1 \mathrm{~atm}$.

\subsection{Effect of the void fraction and role of field bubble compressibility on the primary bubble dynamics}

This section considers the effects of the initial bubbly medium gas volume fraction on the dynamics of the primary bubble and on the flow field it generates. Figure 12 
presents temporal variations of the radius of the primary bubble for different initial void fractions, $\alpha_{0}$. The figure shows the results with the time divided by the bubble period in the pure liquid to clearly bring out the period shortening with an increase in the void fraction. The figure also shows that the amplitude of the oscillations of the primary bubble is noticeably affected by the presence of the surrounding bubbles. This results in noticeable reduction of the bubble volume oscillations (smaller maximum equivalent radius and larger minimum equivalent radius) and similarly in measureable reduction of the bubble period. These reductions have been also observed experimentally (Jayaprakash et al., 2011). This effect is due to exchange of energy between the primary bubble and the surrounding bubbles. The energy is absorbed by these surrounding bubbles and is then released as pressure fluctuations when these bubbles oscillate in turn in response to the excitation, as seen in Figure 9.

The maximum radius and the period reduction of the primary bubble oscillations are directly related to the changes in the compressibility of the bubbly mixture surrounding the bubble due to the presence and behavior of the field bubbles. An indication of this dependence is the direct link between these changes and the initial void fraction, $\alpha_{0}$. This argument can be further strengthened by considering the results shown in Figure 13. In this figure we compare computations where the full two-way interactions are considered with the case where, artificially, only field bubble motion is allowed, while the bubble radius is held constant. Figure 13 shows that the effect of the bubbly medium on the primary bubble amplitude becomes negligible when the surrounding field bubbles are prevented from changing size, i.e., when Equations (5) is not solved. Similarly, a reduction of the period of the primary bubble occurs only when the field bubbles in the mixture are allowed to change volume in response to the primary bubble excitation. 


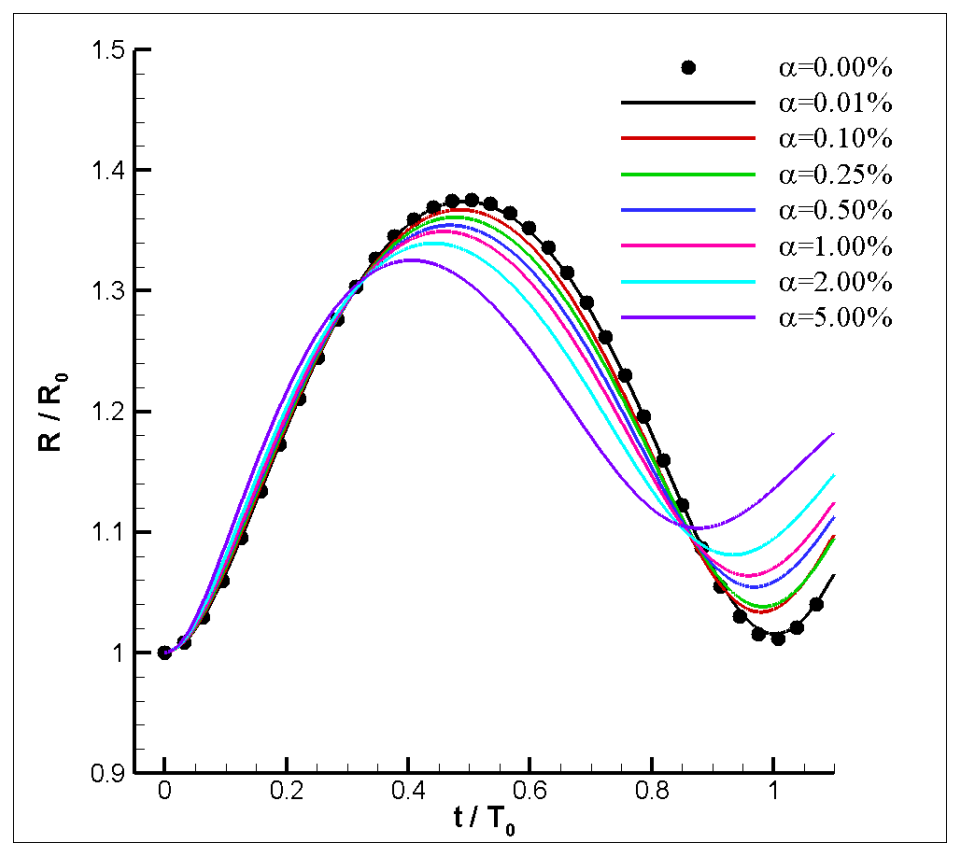

Figure 12: Primary bubble radius versus time divided by $T_{0}$, the computed bubble period in pure water, for different initial void fractions in the bubbly medium surrounding the primary bubble. $R_{0}=5 \mathrm{~mm}, P_{g 0}=2 \mathrm{~atm}, a_{0}=1 \mathrm{~mm}, P_{a m b}=1 \mathrm{~atm}$.

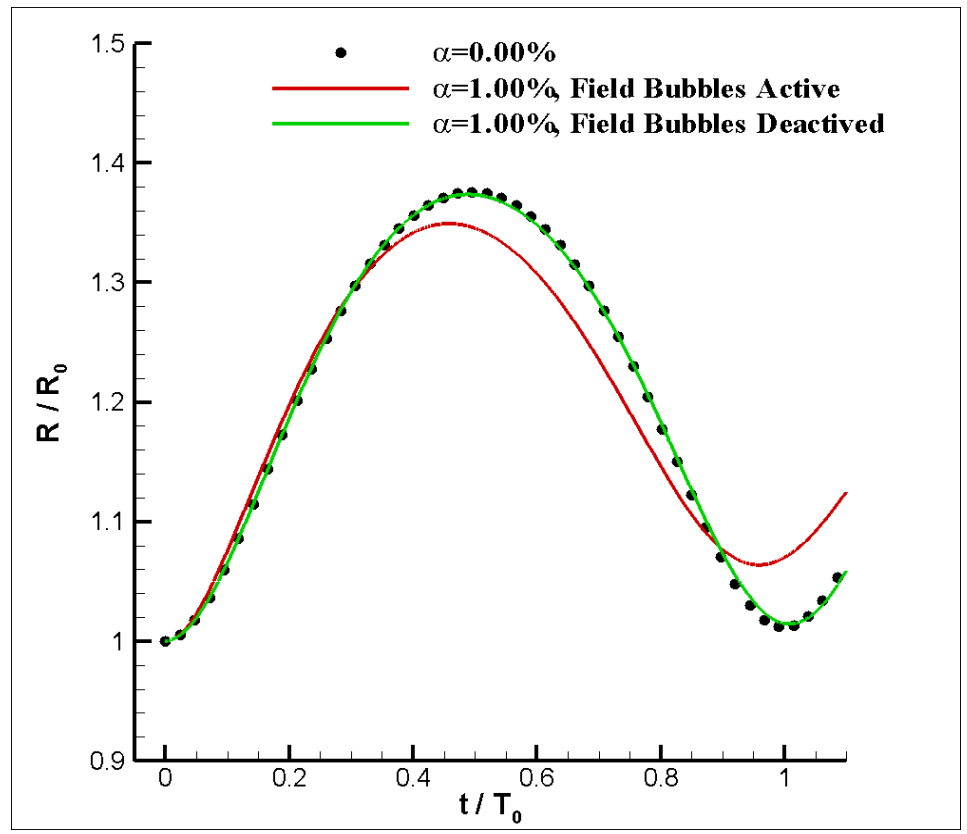

Figure 13: Comparison of the primary bubble radius versus time divided by $T_{0}$, the computed bubble period in pure water, in the pure liquid, and in a bubbly medium with $\alpha_{0}=1.0 \%$ between the full two-way interaction model and when the size change of the field bubbles is artificially turned off. $R_{0}=5 \mathrm{~mm}, P_{g 0}=2 \mathrm{~atm}, a_{0}=1 \mathrm{~mm}, P_{a m b}=1 \mathrm{~atm}$. 


\subsection{Pressure and density variations in the neighborhood of the primary bubble}

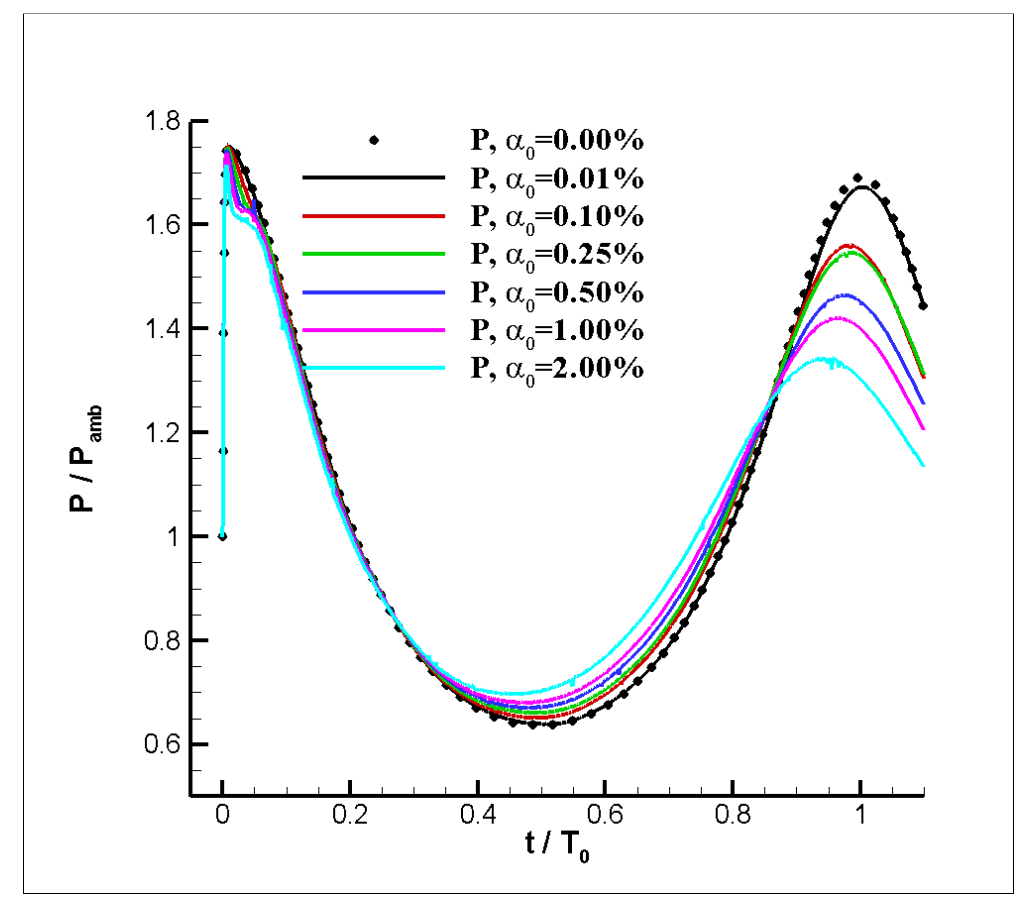

Figure 14 : Comparison of the pressure at $r=7 \mathrm{~mm}\left(r=1.4 R_{0}\right)$ under different initial void fractions. $a_{0}=1 \mathrm{~mm}, R_{0}=5 \mathrm{~mm}, P_{g 0}=2 \mathrm{~atm}, P_{a m b}=1 \mathrm{~atm}$.

As already seen in Figure 11, field bubbles close to the primary bubble respond strongly to the growth and collapse of the primary bubble. Thus, it is of particular interest to further examine their effects on the solution by examining the variations of the pressure and mixture density in the neighborhood of the primary bubble.

Figure 14 shows the pressure time variations at $r=7 \mathrm{~mm}\left(1.4 R_{0}\right)$ for different initial void fractions $\alpha_{0}$. At this location close to the primary bubble wall, the flow feels the high pressures generated by the primary bubble very quickly after the bubble wall is allowed to move. For all cases, the pressure first rises from $1 \mathrm{~atm}$. to a value close to the pressure inside the primary bubble then starts to decrease until it reaches a minimum, corresponding to the primary bubble reaching its maximum value. Then, the pressure gradually increases as the primary bubble collapses following the same pressure versus time shape as the primary bubble volume versus time. The maximum pressure reached at the end of the cycle is however smaller than that of the first peak when the primary 
bubble was released because the bubbly mixture attenuates the pressures with the field bubbles absorbing energy from the main bubble. Figure 14 shows clearly this attenuation increasing for larger void fractions through an increase in the number density of field bubbles (since the field radii have been selected to be the same).

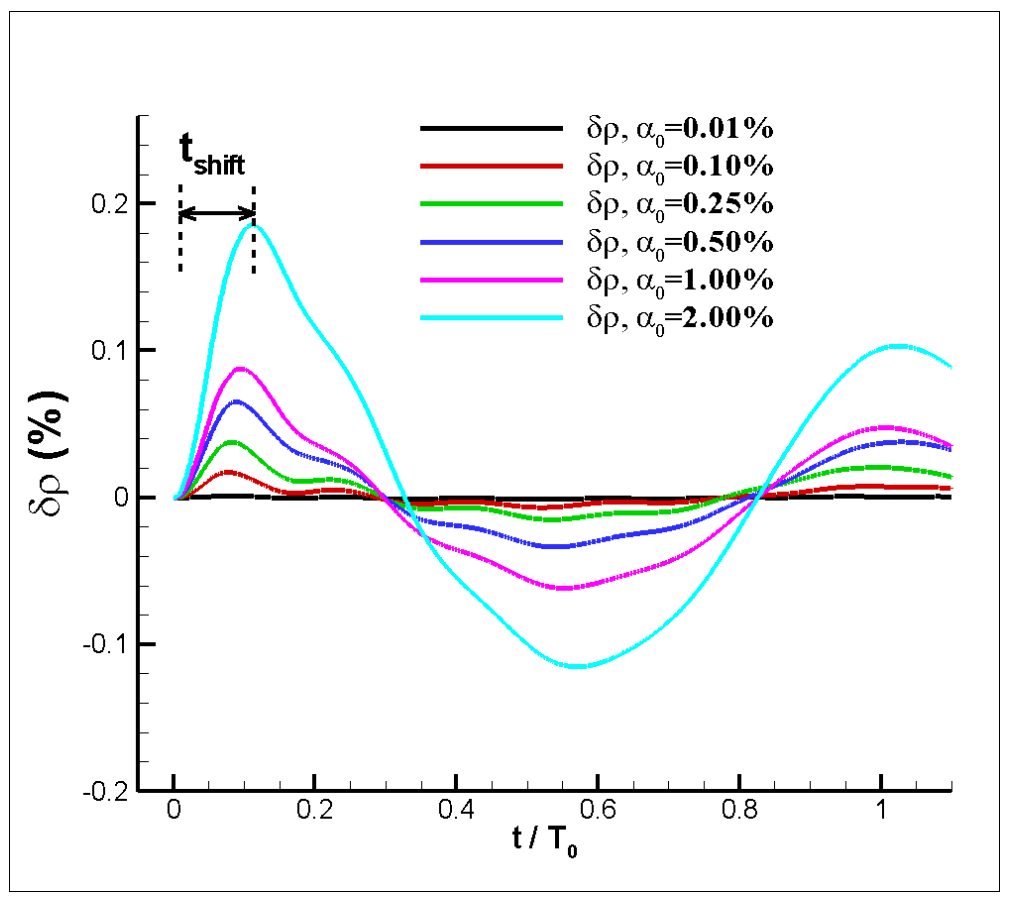

Figure 15: Comparison of the time variations of the relative change, $\delta \rho=\left(\rho_{m}-\rho_{m 0}\right) /$ $\rho_{m 0}$, of the mixture density at $r=7 \mathrm{~mm}\left(r=1.4 R_{0}\right)$ under different initial void fractions. $a_{0}=$ $1 \mathrm{~mm}, R_{0}=5 \mathrm{~mm}, P g_{0}=2 \mathrm{~atm}, P_{a m b}=1 \mathrm{~atm}$.

The corresponding time variations of the mixture densities for various initial void fractions, $\alpha_{0}$, are displayed in Figure 15. Here, the temporal variation of the mixture density, $\delta \rho$, is defined as the relative local mixture density variation compared to the initial mixture density, i.e., $\delta \rho=\left(\rho_{m}-\rho_{m 0}\right) / \rho_{m 0}$. Since the density is linearly related to the void fraction through Equation (1), which in turn is determined by the local pressure, the mixture density oscillates with a period close to that of the primary bubble for all cases. However, a time delay in the occurrence of the first density peak can be clearly seen in the figure and increases with the void fraction. This peak density is also shifted 
relative to the peak in the pressure where the first pressure peak occurs very close to $\mathrm{t}=0$ (see Figure 14). This delay, defined as " $t_{\text {shift }}$ ", is about $7 \%$ of the primary bubble period for $\alpha_{0}=0.1 \%$ and increases to about $13 \%$ for $\alpha_{0}=2 \%$. This is attributed to the two-way interactions between the main bubble dynamics and the bubbles in the flow field. The field bubbles take time to react to the pressure change. This results in finite time of transfer of the density information between the primary bubble and a field point.

Figure 16 compares the temporal variations of the gas pressure inside a field bubble located close to the primary bubble and the two-phase field pressure it encounters. The figure shows that as the primary bubble expands, the pressure around it first rises to a large value and then decreases in time. As long as this pressure is higher than the sum of the pressure of the gas inside a field bubble plus the vapor pressure minus the surface tension, this field bubble continues to lose volume. The gas inside it is compressed until time $t_{\text {shift }}$, when the inner bubble pressure balances the field pressure. As a result, during the time $\left[0, t_{\text {shift }}\right]$, the field bubbles in the considered region get compressed and the mixture density increases, even while the corresponding field pressure decreases. Therefore, the $p$ - $\rho$ phase shift can be attributed to the dynamics of the field bubbles in the two-phase medium, which respond to the field pressure changes slower than the liquid flow does. The phase shift becomes larger as the void fraction increases and as the effect of the field bubbles' dynamics on the primary bubble increases. Further indications supporting this explanation can be seen in the following section where $t_{\text {shift }}$ is found to become larger as the size of the field bubbles increases and as the natural period of oscillation of the bubble increases. This finding cannot be captured by methods assuming homogeneous medium, e.g., (Wardlaw \& Luton, 2000, Wardlaw \& Luton, 2003) and the analytical Gilmore equation (Gilmore, 1952). This highlights the importance of considering the individual bubble dynamics in the dispersed phase for high fidelity modeling. 


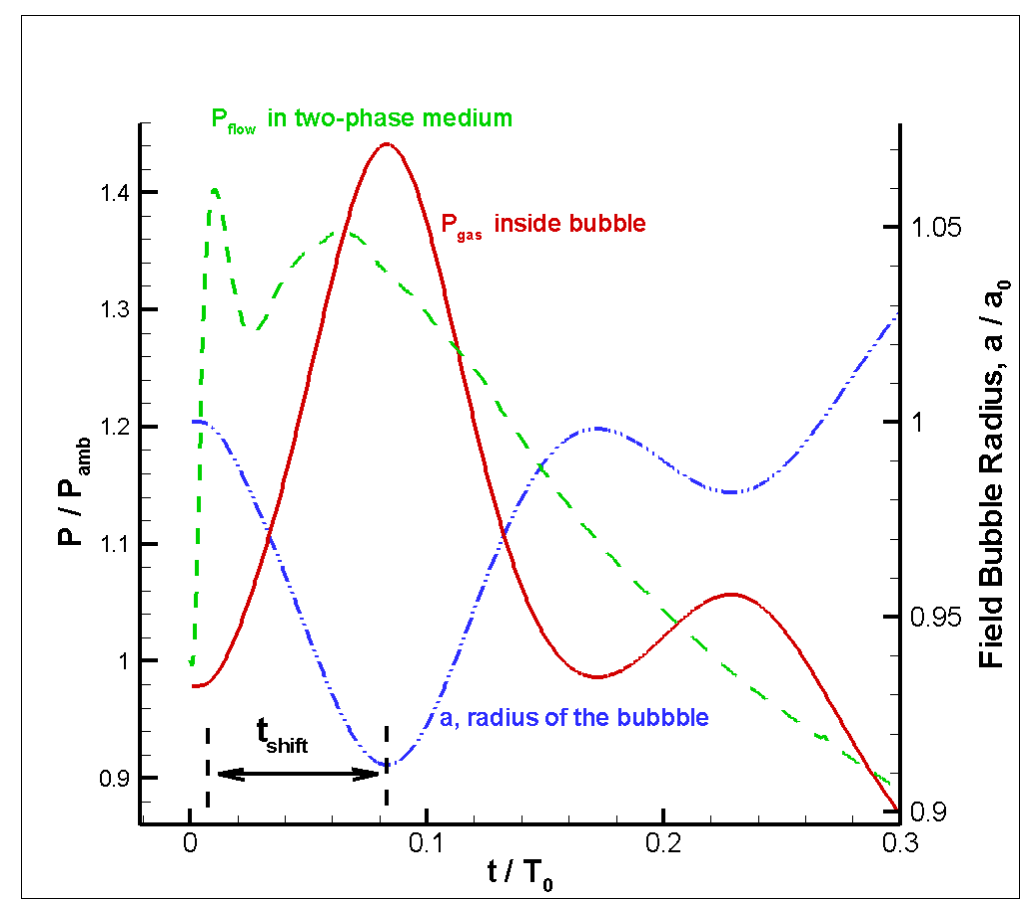

Figure 16: Variations of the gas pressure inside a field bubble and the corresponding encounter pressure in the two-phase medium for a bubble at a distance of $2.8 R_{0}$ from the primary center. $\alpha_{0}=1 \%$. $R_{0}=5 \mathrm{~mm}, P_{g 0}=2 \mathrm{~atm}, a_{0}=1 \mathrm{~mm}, P_{a m b}=1 \mathrm{~atm}$.

\subsection{Effect of field bubbles size and concentration on primary bubble dynamics}

We have illustrated in the previous sections that the dynamics of the primary bubble is significantly modified by the presence of the dispersed bubbles in the two-phase medium and have seen that this effect is dependent on the void fraction. To gain further understanding we consider now the effects of changing the size of the field bubbles. We consider field bubbles with initial radii between one tenth and one fifth of the radius of the primary bubble and consider cases with initial void fractions, $\alpha_{0}$, between $0 \%$ and $5 \%$. 


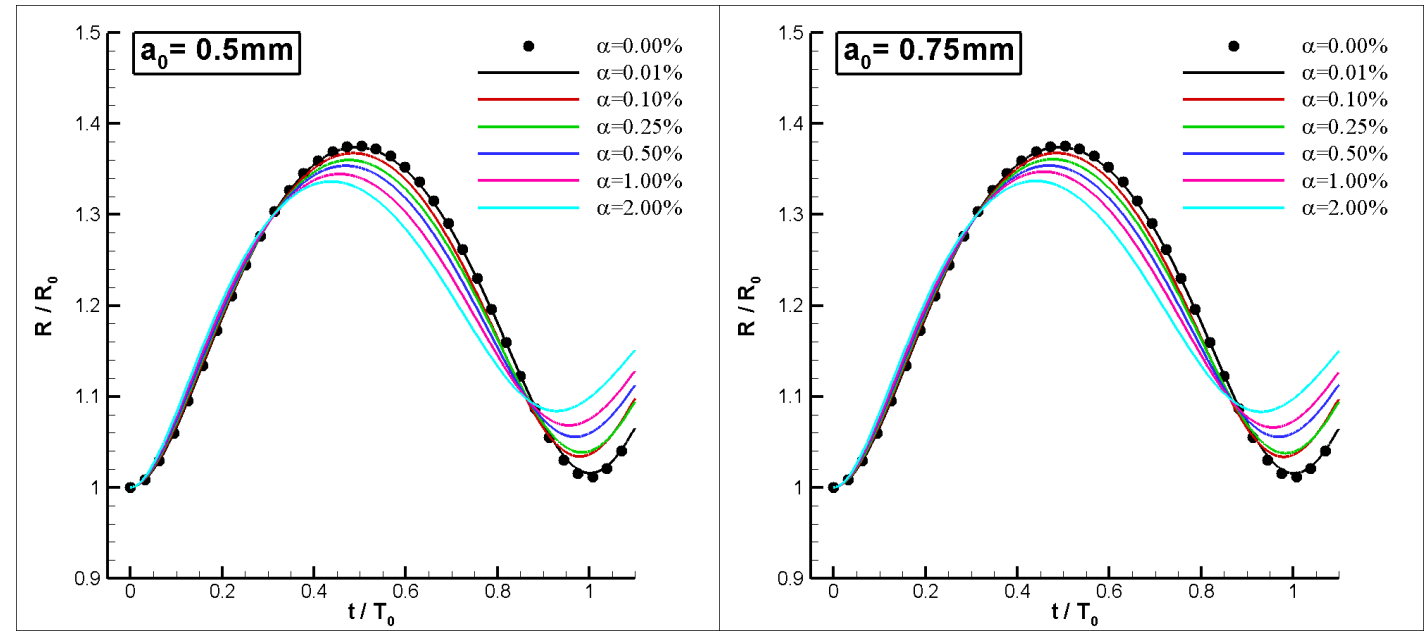

Figure 17: Primary bubble radius versus time for two initial field bubble radii $\left(a_{0}=0.5 \mathrm{~mm}=0.1 R_{0}\right.$, and $\left.a_{0}=0.75 \mathrm{~mm}=0.15 R_{0}\right)$ and for a set of initial void fractions between 0 and $2 \% . R_{0}=5 \mathrm{~mm}, P_{g 0}=2 \mathrm{~atm}, P_{a m b}=1 \mathrm{~atm}$.

Figure 17 compares the variations of the primary bubble radius versus time for different void fractions and for two initial radii. Overall, as in Figure 12, the results show that as the void fraction increases, the maximum bubble radius decreases while the minimum bubble radius increases, i.e. the amplitude of the bubble radius oscillations are significantly reduced. This is in a good agreement with the solutions of analytical Gilmore equation. However, the Euler-Lagrange modeling shows less reduction in the bubble oscillations amplitude than with the Gilmore model and the difference becomes more and more obvious for increasing $\alpha_{0}$. This is illustrated in Figure 18a, which shows the maximum and minimum bubble radii achieved for different void fractions normalized with the values in pure water. These are shown for three initial bubble radius values. The figure also shows the results obtained from the Gilmore model. A significant effect of the void fraction can be seen and differences between the present results and the homogeneous model are seen to increase with the void fraction. Much less influence is seen for the initial bubble radius. Under the same initial void fraction, damping of the oscillations of the primary bubble is a little larger for smaller field bubbles size. For example, at $\alpha_{O} \sim 1 \%$, the reduction of $R_{\max }$ is about $2.3 \%$ for field 
bubbles of radii $r_{\sigma}=0.5 \mathrm{~mm}$ and $2.1 \%$ for $a_{0}=0.75 \mathrm{~mm}$, but this drops to $1.9 \%$ for field bubbles of radii $a_{0}=1 \mathrm{~mm}$.

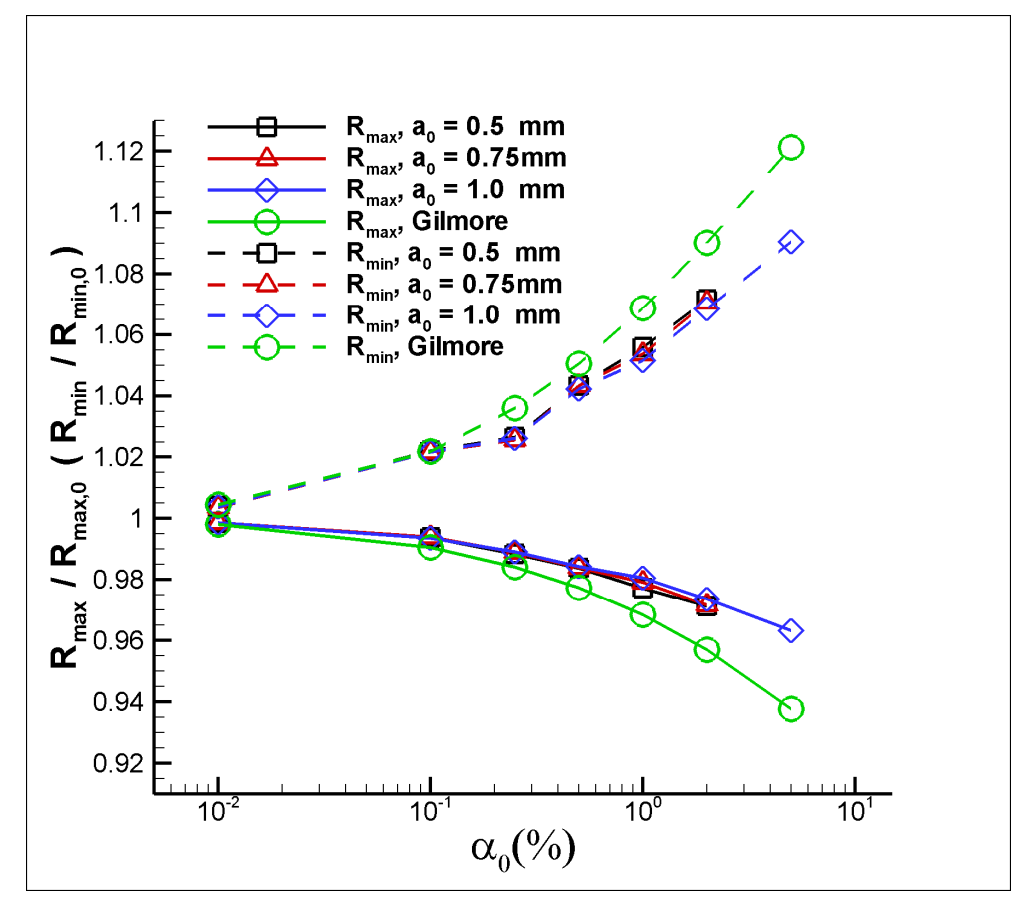

Figure 18: Variation with the void fraction of the maximum and minimum radii, $R_{\max }$ and $R_{\min }$, relative to the same in the pure liquid for three initial field bubble radii ( $a_{0}=0.5 \mathrm{~mm}=0.1 R_{0}$, $a_{0}=0.75 \mathrm{~mm}=0.15 R_{0}$, and $a_{0}=1.0 \mathrm{~mm}=R_{0} / 5$,) and for a set of initial void fractions between 0 and $5 \% . R_{0}=5 \mathrm{~mm}, P_{\mathrm{g} 0}=2 \mathrm{~atm}, P_{a m b}=1 \mathrm{~atm}$.

The reduction of damping with increasing void fraction relative to the Gilmore model can be examined by considering the variations in the bubble radius time dependence as the void fraction is increased. As seen in Figure 19, the bubble at $r=$ $4 R_{0}$ oscillates at a frequency composed of the primary bubble forcing frequency and its own natural frequency These oscillations absorb energy from the primary bubble and from each other, and are responsible for the damping effects onto the primary bubble dynamics and onto each other. This effect increases as the void fraction increases. Changes in the void fraction are fed-back to the mixture flow through modification of the mixture density and sound speed. The modified properties, in turn, affect the dynamics of the field bubbles by reducing the driving pressure for each bubble. This is 
reflected in Figure 19, which shows smaller amplitude of the oscillations of the field bubble size as the void fraction increases, and made further clear in Figure 20, which compares the amplitudes of the secondary oscillation for the different initial void fractions. This effect is actually self-limiting. As the void fraction increases, less energy is transmitted to the field bubbles and they in turn have less chance to absorb the energy. The above discussion can be helped by also comparing in Figure 19 the above discussed field bubble oscillations with those obtained when imposing only one-way coupling (i.e., the mixture flow does not see the presence of the field bubbles and has the same properties as a pure liquid). In this case the field bubbles size oscillates with a much larger amplitude than when the correct two-way coupling is applied. Obviously, in the Gilmore solution, these damping effects happening between the field bubbles due to their own interaction are not included, thus resulting in over predicted energy reduction effects on the primary bubble dynamics.

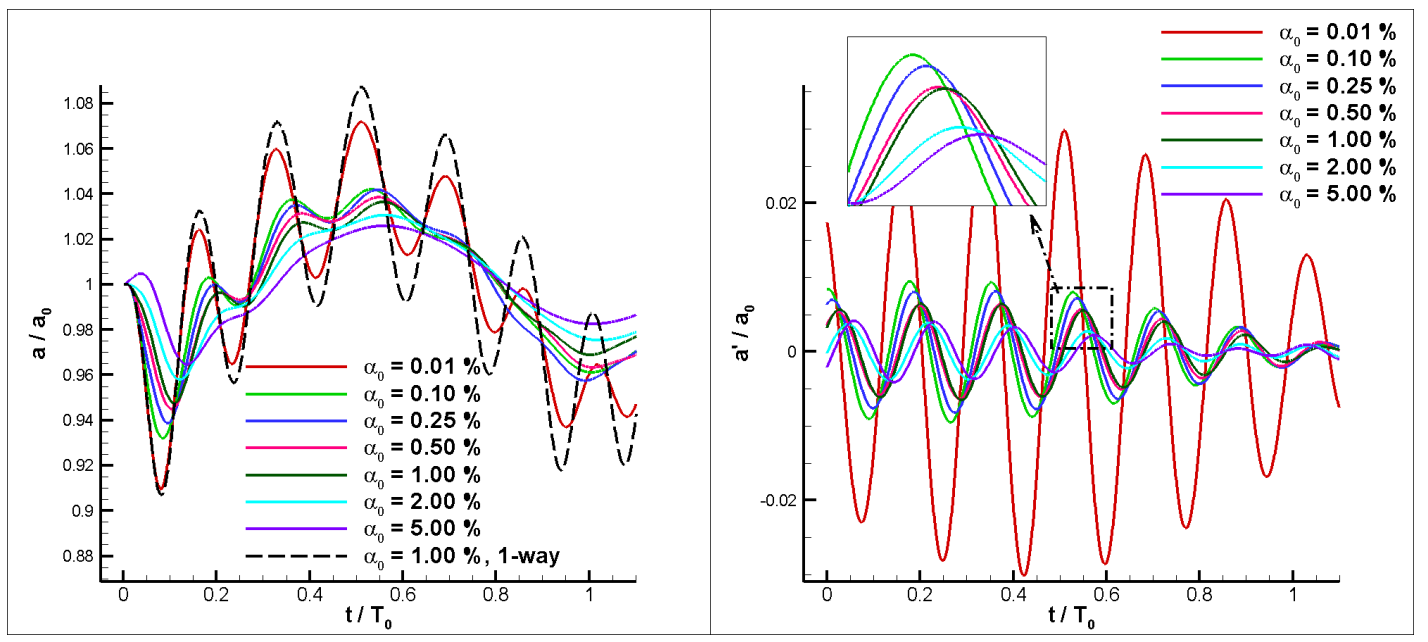

Figure 19: (Left) Comparison of the radius versus time of a field bubble initially at $r=4 R_{0}$ for different initial void fractions in the mixture. Note the reduction in response as the void fraction increases. $a_{0}=1 \mathrm{~mm}, R_{0}=5 \mathrm{~mm}, P_{g 0}=2 \mathrm{~atm}, P_{a m b}=1 \mathrm{~atm}$. (Right) Filtered radius variation, $a$ versus time for the same bubbles in left, where $a$ is the amplitude of the secondary bubble oscillations under their own natural frequency. 


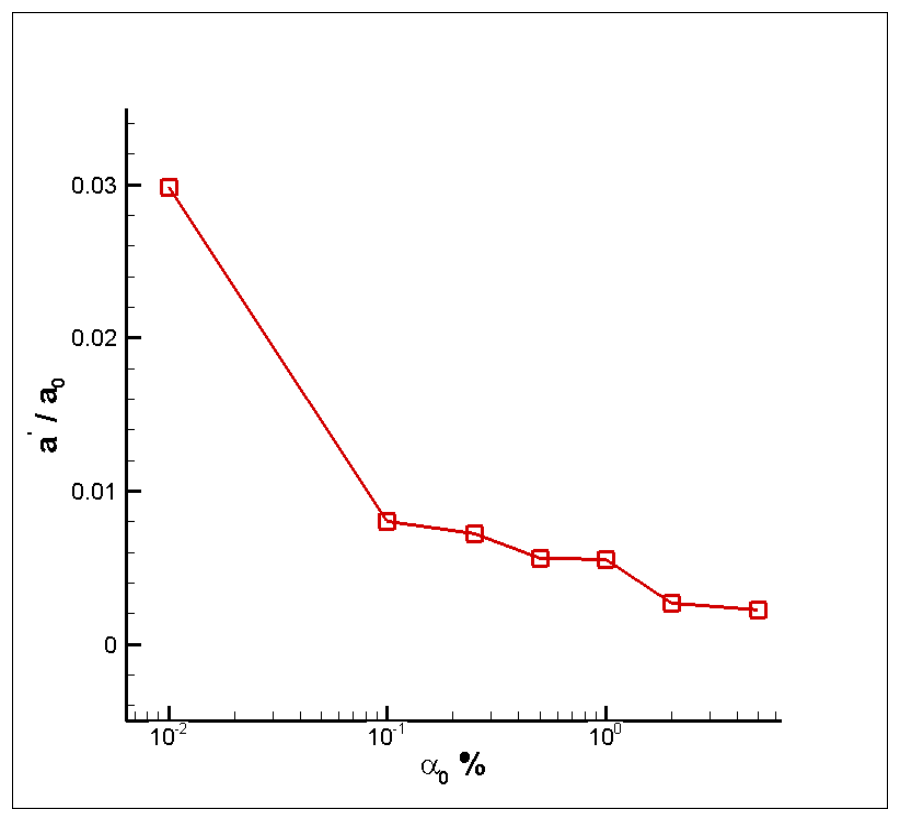

Figure 20: Effect of the void fraction on the amplitude of the secondary oscillations at the field bubble resonance frequency, a', shown in above right plot of Figure 19.

Similarly, the trend of reduced damping when increasing the size of the field bubbles can be explained by considering the field bubble radius oscillations in Figure 21. A field bubble of different sizes located at $r=4 R_{0}$ is considered for the same $\alpha_{0}=1.0 \%$. Though overall, the bubble sizes oscillate with similar trends, by increasing the size of the field bubbles we bring their natural frequency of oscillations closer to that of the primary bubble and the frequency of the secondary oscillations is reduced. This results in less energy dissipation and weaker local pressure perturbations than with the high frequency oscillations of the smaller field bubbles. As a result, as shown in Figure 22 with a same initial void fraction $\alpha_{0}$, the attenuation of pressure becomes slightly weaker as the size of the field bubbles increases.

Figure 23 shows the effect of the presence of the field bubbles on the period of the primary bubble. The results are normalized by the bubble period in a pure liquid (i.e. at $\alpha_{0}=0$ ) to illustrate the reduction in the period when in a two-phase medium. The period reduction effect is seen to increase with the void fraction and to not depend much on the field bubbles size. This numerically computed effect is much larger than that estimated 
using the Gilmore equation and other analytical expressions assuming homogenous flows. For example, if we consider that the period reduction is only due to a reduction in the mixture density, the Rayleigh time would be modified as

$$
T_{\text {Rayleigh, } \alpha_{0}}=2 R_{0} \sqrt{\frac{\rho_{l}\left(1-\alpha_{0}\right)}{P_{g 0}-P_{a m b}} .}
$$

Accordingly a bubbly mixture with $\alpha_{0}=5 \%$ would result in about $2.2 \%$ reduction in the period; however, it is around 13\% in Figure 23. This significant difference should be attributed to the interactive dynamics between the field bubbles and the effect of their response on the primary bubble dynamics, which are accounted for in the present simulations.

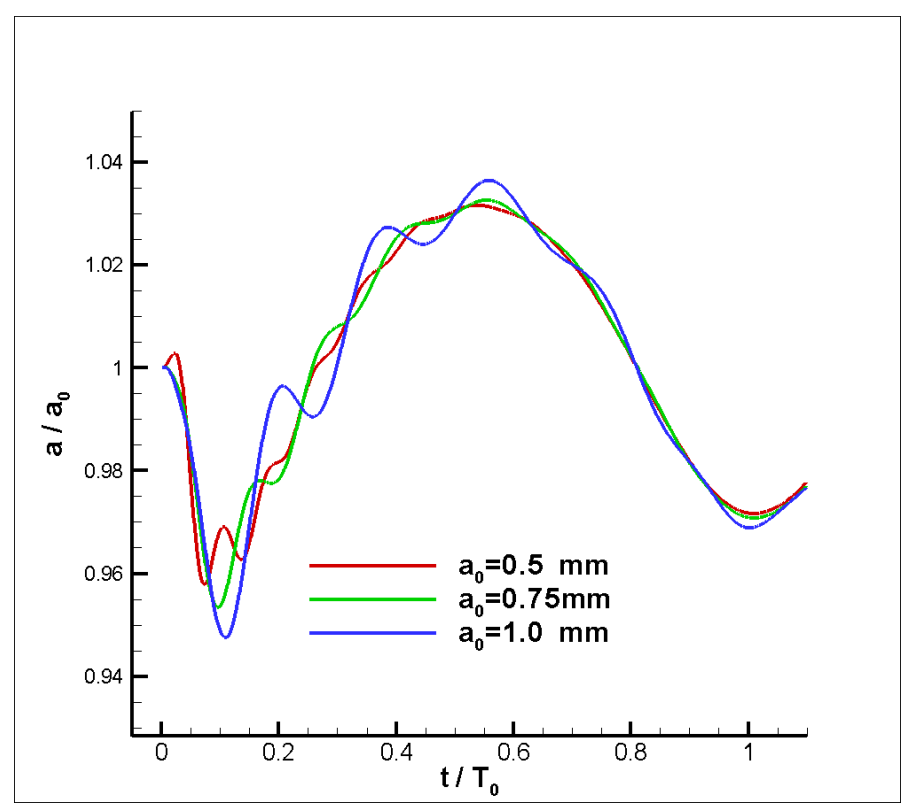

Figure 21: Comparison of the radius versus time of a field bubble initially at $r=4 R_{0}$ for different initial bubble sizes. $\alpha_{0}=1.0 \%, R_{0}=5 \mathrm{~mm}, P_{g 0}=2 \mathrm{~atm}, P_{a m b}=1 \mathrm{~atm}$. 


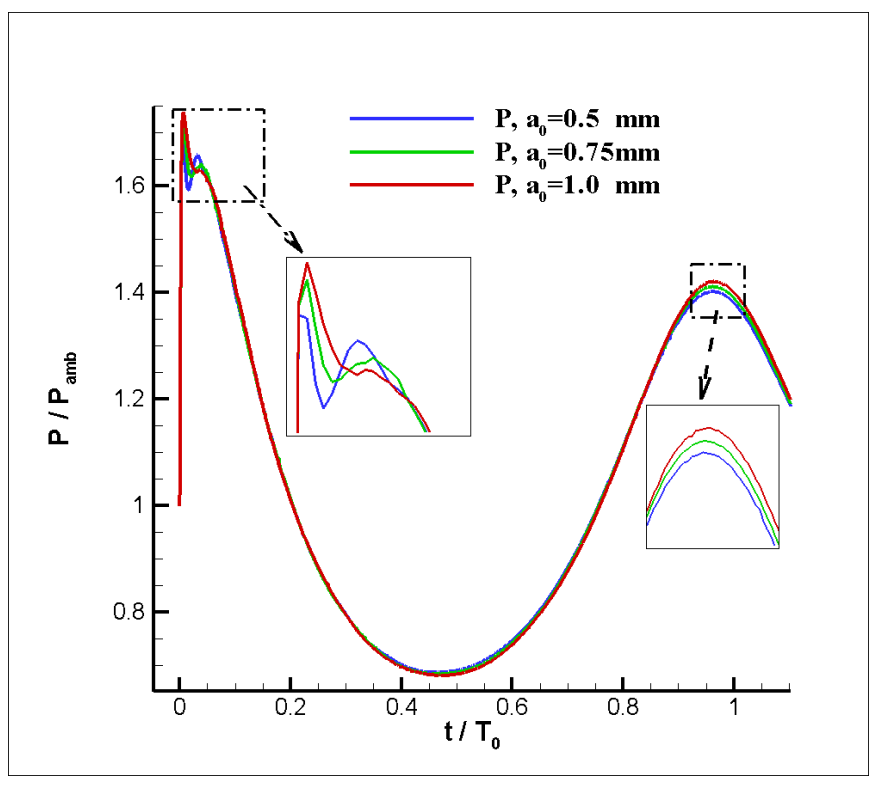

Figure 22: Comparison of the pressure at $\mathrm{r}=7 \mathrm{~mm}$ with different initial field bubble sizes. $\alpha_{0}=1.0 \%, R_{0}=5 \mathrm{~mm}, P_{g 0}=2 \mathrm{~atm}, P_{a m b}=1 \mathrm{~atm}$.

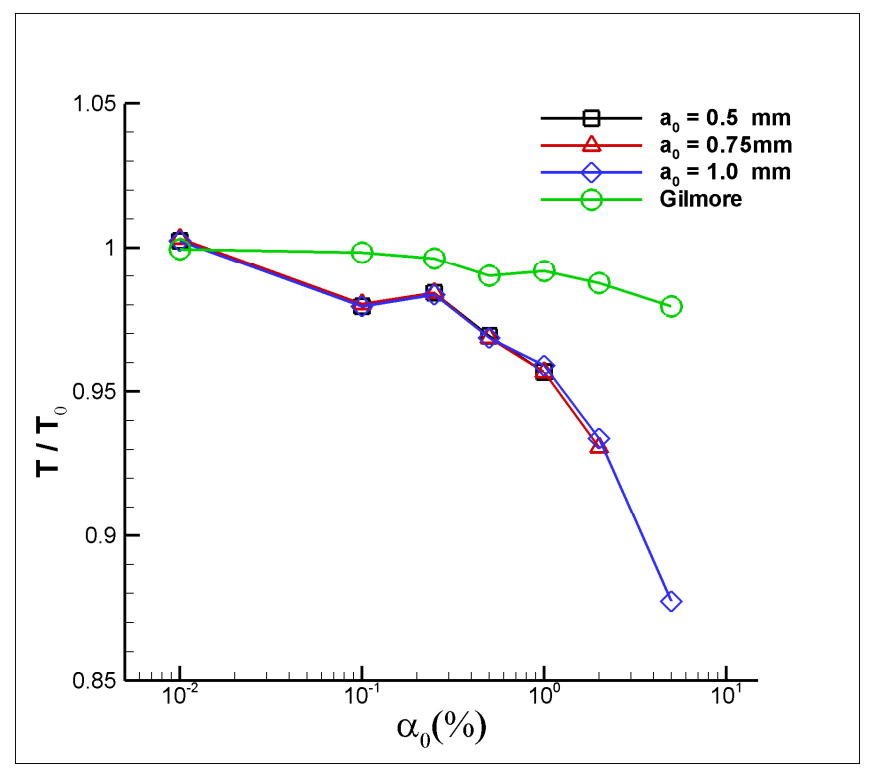

Figure 23: Comparison of the bubble period achieved by the primary bubble for three initial field bubble radii $\left(a_{0}=0.5 \mathrm{~mm}=0.1 R_{0}, 0.75 \mathrm{~mm}=0.15 R_{0}\right.$, and $1.0 \mathrm{~mm}=R_{0} / 5$, ) and for a set of initial void fractions between 0 and $5 \% . R_{0}=5 \mathrm{~mm}, P_{g 0}=2 \mathrm{~atm}, a_{0}=0.5 \sim 1 \mathrm{~mm}, P_{\text {amb }}=1 \mathrm{~atm}$. 


\subsection{Effect of field bubbles size and concentration on Phase shift}

Here we consider the effect of the field bubbles on the normalized mixture density change $\delta \rho=\left(\rho_{m}-\rho_{m 0}\right) / \rho_{m 0}$ and on the pressure distribution. Figure 24 displays the curves of mixture density versus time monitored at a point in the near field of the primary bubble $\left(r=7 \mathrm{~mm}=1.4 R_{0}\right)$ under the different conditions used above. Overall, all cases show that as the void fraction increases the phase shift becomes more and more significant due to the increased effect of the field bubbles on the primary bubble. However, this effect depends on both the bubble sizes and the void fraction. Figure 25 quantitatively compares $t_{\text {shift }}$ for the different conditions to illustrate this effect. The Gilmore equation does not capture $t_{\text {shift }}$ due to the homogenous medium assumptions where the mixture density is only a function of the pressure without consideration to bubble dynamics in the field. We can see in the figure that for a given initial bubble size, $t_{\text {shift }}$ becomes larger as the void fraction increases. However, the effect of the field bubbles sizes for the same $\alpha_{0}$ is more complex. As seen in Figure 25, a switch in behavior occurs as the void fraction increases. At small void fractions, $t_{\text {shift }}$ becomes larger as the initial bubble radius increases. This is due to the fact that the natural bubble period increases with the size. Therefore, these bubbles take a longer time to reach balance with the flow. This is also clearly seen in the bubble size variation history curves plotted in Figure 21, where we see an initial stage of bubble size decrease (mixture density increasing) while the pressure is also dropping. The duration of this stage is longer for the larger bubbles. However, this trend reverts for the higher void fraction, here for $\alpha_{0}>2 \%$. This can be explained by the same reasoning given previously about increased damping and reduced secondary oscillations of the field bubble at their own natural frequency as the void fraction increases. 

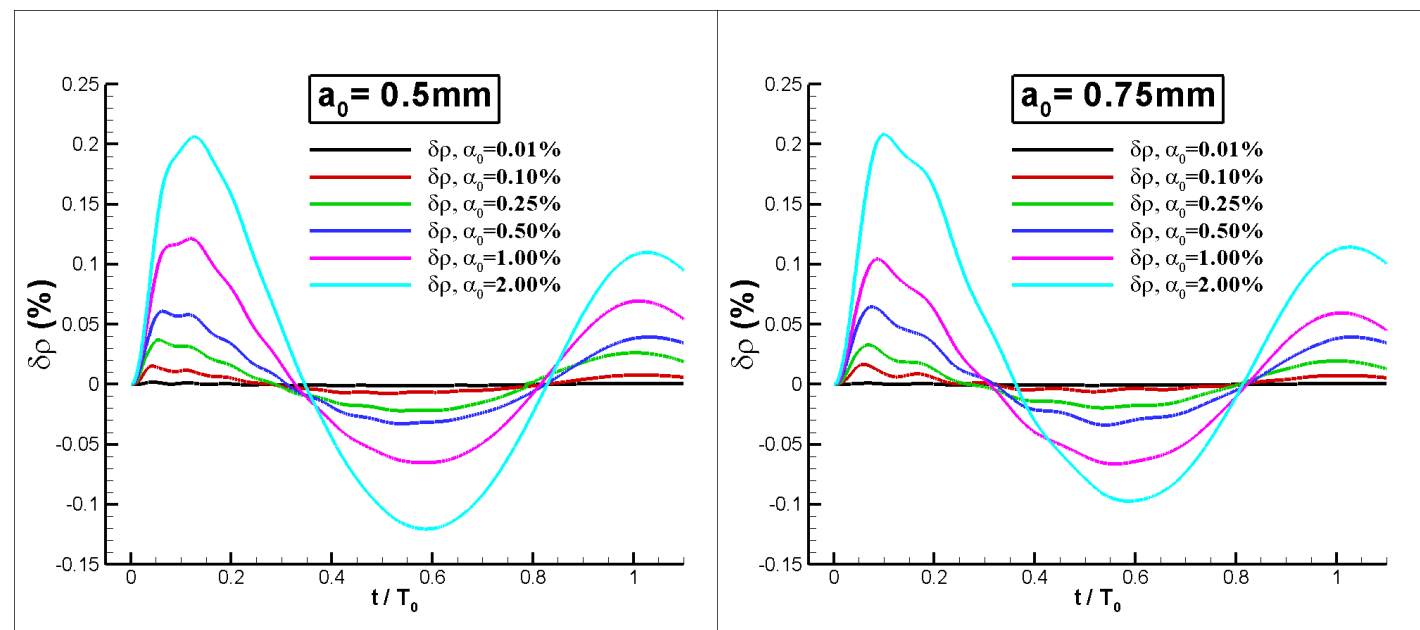

Figure 24: Variation of the relative change of the mixture density, $\delta \rho=\left(\rho_{m}-\rho_{m 0}\right) / \rho_{m 0}$, at a radial distance of $r=7 \mathrm{~mm}$ for two initial field bubble radii $\left(a_{0}=0.5 \mathrm{~mm}=0.1 R_{0}\right.$, and $a_{0}=0.75$ $m m=0.15 R_{0}$ ) and for a set of initial void fractions between 0 and $5 \% . R_{0}=5 \mathrm{~mm}, P_{g 0}=2 \mathrm{~atm}$, $a_{0}=0.5 \sim 1 \mathrm{~mm}, P_{a m b}=1 \mathrm{~atm}$. (Refer to above Figure 15 for comparison with cases of $a_{0}=1.0$ $\left.m m=R_{0} / 5\right)$

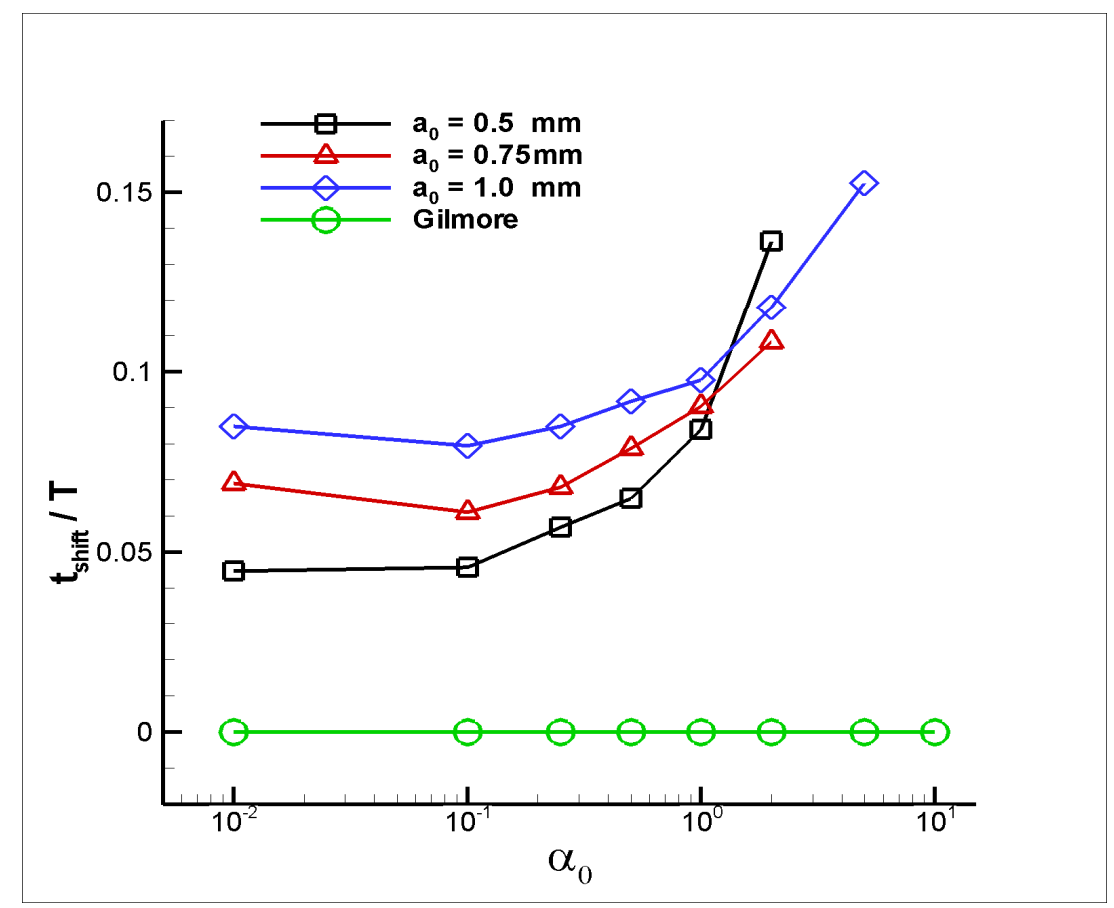

Figure 25: Comparison of the phase shift between pressure and density, $t_{\text {shift }}$, for three initial field bubble radii $\left(a_{0}=0.5 \mathrm{~mm}=0.1 R_{0}, a_{0}=0.75 \mathrm{~mm}=0.15 R_{0}\right.$, and $\left.a_{0}=1.0 \mathrm{~mm}=R_{0} / 5\right)$ and for a set of initial void fractions between 0 and $5 \% . R_{0}=5 \mathrm{~mm}, P_{g 0}=2 \mathrm{~atm}, a_{0}=0.5 \sim 1 \mathrm{~mm}, P_{a m b}=$ $1 \mathrm{~atm}$. 


\section{CONCLUSIONS}

The dynamics of a primary bubble oscillating in a bubbly flow is studied using a 3D two-way coupled Euler-Lagrange model, which treats the two-phase medium as a continuum while the field bubbles are tracked in a Lagrangian fashion. The two-phase medium conditions were varied by changing the size and number of the field bubbles in the two-phase medium. This modified also the initial void fractions.

The study has shown that bubbly dynamics is modified as follows in a bubbly medium. The surrounding bubbly medium absorbs the energy emitted from the primary bubble thereby damping the bubble oscillations and resulting in noticeable reductions in both the maximum achieved primary bubble radius and bubble period. These effects become more significant when the void fraction of the mixture increases.

Under the same void fraction, smaller size field bubbles in the two-phase medium (or alternatively larger ratio of primary bubble to surrounding bubbles) result in stronger damping effects in terms of achieved maximum radius of the primary bubble. However, varying the field bubble size (or alternatively changing the ratio of primary bubble to surrounding bubbles) does not seem to have a noticeable effect on the primary bubble period. This implies that for developing a correction term for the homogeneous model, it will be possible to correct the bubble period based on the mixture void fraction. However, more work is needed to correct the amplitude as there is a need to study this further for cases where the primary bubble becomes in the same range and even smaller than the surrounding field bubbles.

On the other hand, the dynamics of the field bubbles result in a phase-shift between the density and the pressure time variations in the two-phase medium and this is strongly influenced by the bubble sizes and the void fraction. The effects cannot be captured by analytical solutions treating the mixture as a homogeneous medium. The phase shift increases with the void fraction but is a more complex function of the size of the dispersed small bubbles. At smaller void fractions larger bubbles produce larger phase shifts but this trend is revered at the higher void fractions. Here again, for future development of corrections for the homogeneous model, more work is needed to 
address cases where the primary bubble becomes in the same range and even smaller than the surrounding field bubbles. Also, investigating how the pressure ratio between the initial pressures inside the primary and field bubbles affects the differences between the homogeneous models and the present model will provide insight to the development of a correction strategy.

\section{ACKNOWLEDGMENTS}

This work was supported by the Department of Energy under contract DE-FG0207ER84839. This work was also supported in part by grants of computer time from The National Energy Research Scientific Computing Center (NERSC), a scientific computing facility for the Office of Science in the U.S. Department of Energy.

\section{NOMENCLATURE}

$C_{D} \quad$ Drag coefficient

$C_{L} \quad$ Lift coefficient

$\mathrm{F}_{\mathrm{r}} \quad$ Froude number

$P \quad$ Pressure at bubble wall

$P_{a m b} \quad$ Ambient Pressure

$P_{\infty} \quad$ Pressure at infinity

$r \quad$ Radial location

$a_{0} \quad$ Initial bubble size in bubbly medium

$R \quad$ Primary bubble radius

$R_{\max } \quad$ Maximum radius of primary bubble

$R_{e} \quad$ Reynolds number

$U, V, W \quad$ Contravariant velocity components

$\mathrm{W}_{\mathrm{e} b} \quad$ Weber number

$c \quad$ Sound speed in the bubbly medium

$c_{\infty} \quad$ Reference sound speed

g Acceleration of gravity vector

$k \quad$ Polytropic gas constant

$p \quad$ Pressure 


$\begin{array}{ll}p_{v} & \text { Vapor pressure } \\ t & \text { Time } \\ t_{\text {shift }} & \text { Phase shift between the mixture density and pressure variations } \\ T_{0} & \text { Duration of the first cycle of oscillations of the primary bubble } \\ u & \text { Velocity } \\ u_{c h a r c} & \text { Characteristic velocity } \\ V & \text { Volume } \\ \alpha & \text { Void fraction } \\ \beta_{c} & \text { Artificial compressibility factor } \\ \gamma & \text { Surface tension at air-water interface } \\ \omega & \text { Vorticity } \\ \rho_{m} & \text { Density of the mixture } \\ \delta \rho & \text { Variation of mixture density } \\ \rho_{\infty} & \text { Reference density } \\ \mu & \text { Dynamic Viscosity } \\ v & \text { Kinematic Viscosity } \\ \tau & \text { Pseudo-time } \\ \xi, \eta, \zeta & \text { Curvilinear coordinates }\end{array}$

\section{Subscripts}

$0 \quad$ Initial condition

b, bubble Bubble property

enc Encountered by the bubble

$i, j, k \quad$ Indices

$g \quad$ Gas property

$l \quad$ Liquid property

$m \quad$ Mixture property

\section{REFERENCES}

AAnjaneya, M., PATKAR, S. \& FEDKIw, R. 2013. A monolithic mass tracking formulation for bubbles in incompressible flow. Journal of Computational Physics, 247, 17-61.

AliabAdI, A. \& TAKLIFI, A. 2012. The effect of magnetic field on dynamics of gas bubbles in visco-elastic fluids. Applied Mathematical Modelling, 36, 2567-2577.

Anderson, W. K., Thomas, J. L. \& VAn LeER, B. 1986. Comparison of finite volume flux vector splittings for the Euler equations. AIAA journal, 24, 1453-1460. 
Arienti, M. \& Sussman, M. 2014. An embedded level set method for sharp-interface multiphase simulations of Diesel injectors. International Journal of Multiphase Flow, 59, 1-14.

ARnDT, R. E. A. 1981. Cavitation in fluid machinery and hydraulic structures. Annual Review of Fluid Mechanics, 13, 273-328.

Balachandar, S. \& EATON, J. K. 2010. Turbulent dispersed multiphase flow. Annual Review of Fluid Mechanics, 42, 111-133.

BATCHELOR, G. K. 1967. An introduction to fluid dynamics, Cambridge university press.

Besant, W. 1859. Hydrostatics and hydrodynamics. Cambridge University Press, London, Art, 158, 1859.

BJERKNES, V. F. K. 1906. Fields of Force, New York, Columbia University Press.

BlAKE, J. R. \& GIBSON, D. C. 1987. Cavitation bubbles near boundaries. Annual Review of Fluid Mechanics, 19, 99-123.

Brennen, C. E. 1995. Cavitation and bubble dynamics, Oxford University Press

Brennen, C. E., 2005, Fundamentals of Multiphase Flows, Cambridge University Press.

Calvisi, M. L., Lindau, O., Blake, J. R. \& Szeri, A. J. 2007. Shape stability and violent collapse of microbubbles in acoustic traveling waves. Physics of Fluids, $19,047101$.

CAPECElatro, J. \& Desjardins, O. 2013. An Euler-Lagrange strategy for simulating particle-laden flows. Journal of Computational Physics, 238, 1-31.

Capretto, L., Cheng, W., Hill, M. \& Zhang, X. L. 2011. Micromixing Within Microfluidic Devices. Microfluidics: Technologies and Applications, 304, 27-68.

Chahine, G. L., 1983, Cloud Cavitation: Theory. Proc. 14th Symposium on Naval Hydrodynamics, Ann Arbor, Michigan, National Academy Press, pp. 165-194.

Chahine, G. L. and Duraiswami, R., 1992, Dynamical Interaction in a multi-Bubble Cloud. ASME J. Fluids Eng., 114(4), pp. 680-686.

Chahine, G., Duraiswami, R. \& Kalumuck, K. 1996. Boundary element method for calculating 2-D and 3-D underwater explosion bubble loading on nearby structures including fluid structure interaction effects. Naval Surface Weapons Center, Dahlgren Division, Weapons Research and Technology Department, Technical Report NSWCDD/TR-93/46.

Chahine, G. \& KALUMUCK, K. 1998. BEM software for free surface flow simulation including fluid-structure interaction effects. International Journal of Computer Applications in Technology, 11, 177-198.

Chahine, G. L. 2009. Numerical simulation of bubble flow interactions. Journal of Hydrodynamics, Ser. B, 21, 316-332.

Chahine, G. L., Hsiao, C.-T., Choi, J.-K., And Wu, X., 2008, Bubble Augmented Waterjet Propulsion: Two-Phase Model Development and Experimental Validation, 27th Symposium on Naval Hydrodynamics, Seoul, Korea, 5-10, October 2008.

Chahine, G. L. \& HARris, G. 1997. Development and validation of a multicycle bubble model for UNDEX application. 68th Shock and Vibration Symposium. Hunt Valley, MD. 
Chahine, G. L. \& HsiaO, C.-T. Modeling 3D unsteady sheet cavities using a coupled UnRANS-BEM code. In: Proc. 23rd ONR Symposium on Naval Hydrodynamics, 2000.

Chahine, G. L. \& HsiaO, C.-T. 2012. Modeling microbubble dynamics in biomedical applications. Journal of Hydrodynamics, Ser. B, 24, 169-183.

Chahine, G. L. \& LiU, H. L. 1985. A singular-perturbation theory of the growth of a bubble cluster in a superheated liquid. Journal of Fluid Mechanics, 156, 257279.

Chen, J., YAnG, N., Ge, W. \& Li, J. 2009. Computational fluid dynamics simulation of regime transition in bubble columns incorporating the dual-bubble-size model. Industrial \& Engineering Chemistry Research, 48, 8172-8179.

Choi, J., Hsiao, C.-T., Chahine, G. \& Ceccio, S. 2009. Growth, oscillation and collapse of vortex cavitation bubbles. Journal of Fluid Mechanics, 624, 255.

ChORIN, A. J. 1967. A numerical method for solving incompressible viscous flow problems. Journal of computational physics, 2, 12-26.

Chung, S. K. \& CHO, S. K. 2009. 3-D manipulation of millimeter- and micro-sized objects using an acoustically excited oscillating bubble. Microfluidics and Nanofluidics, 6, 261-265.

Coleman, A. J., Saunders, J. E., Crum, L. A. \& Dyson, M. 1987. Acoustic Cavitation Generated by an Extracorporeal Shockwave Lithotripter. Ultrasound in Medicine and Biology, 13, 69-76.

Commander, K. W. \& ProsperetTi, A. 1989. Linear pressure waves in bubbly liquids: Comparison between theory and experiments. The Journal of the Acoustical Society of America, 85, 732.

Darmana, D., DeEn, N. \& KuiPers, J. 2006. Parallelization of an Euler-Lagrange model using mixed domain decomposition and a mirror domain technique: Application to dispersed gas-liquid two-phase flow. Journal of computational physics, 220, 216-248.

Darmana, D., Henket, R., Deen, N. \& Kuipers, J. 2007. Detailed modelling of hydrodynamics, mass transfer and chemical reactions in a bubble column using a discrete bubble model: Chemisorption of $\mathrm{CO} 2$ into $\mathrm{NaOH}$ solution, numerical and experimental study. Chemical Engineering Science, 62, 2556-2575.

DE GraAf, K., Penesis, I. \& BRAndner, P. Comparison of the Rayleigh-Plesset and Gilmore Equations and Additional Aspects for the Modelling of Seismic Airgun Bubble Dynamics. In: 18th Australasian Fluid Mechanics Conference, 2012. 14.

Ferrante, A., AND Elghobashi, S., 2004, On the physical mechanisms of drag reduction in a spatially developing turbulent boundary layer laden with microbubbles, J. of Fluid Mechanics, 503, pp. 345-355.

Francois, M. M., Cummins, S. J., Dendy, E. D., Kothe, D. B., Sicilian, J. M. \& WiLliams, M. W. 2006. A balanced-force algorithm for continuous and sharp interfacial surface tension models within a volume tracking framework. Journal of Computational Physics, 213, 141-173. 
Fuster, D. \& Colonius, T. 2011. Modelling bubble clusters in compressible liquids. Journal of Fluid Mechanics, 688, 352-389.

GeERS, T. L. \& HunTER, K. S. 2002. An integrated wave-effects model for an underwater explosion bubble. The Journal of the Acoustical Society of America, $111,1584$.

GILMORE, F. 1952. The collapse and growth of a spherical bubble in a viscous compressible liquid. Hydrodynamics Lab Report No. 26-4. Pasadena, CA.: Califonia Institute of Technology.

Guillen, D. P., Grimmett, T., Gandrik, A. M. \& Antal, S. P. 2011. Development of a computational multiphase flow model for Fischer Tropsch synthesis in a slurry bubble column reactor. Chemical Engineering Journal, 176, 83-94.

Haberman, W. \& Morton, R. 1953. An experimental investigation of the drag and shape of air bubbles rising in various liquids. DTIC Document.

HERRING, C. 1941. Theory of the pulsations of the gas bubble produced by an underwater explosion, Columbia Univ., Division of National Defense Research.

Hilgenfeldt, S., Brenner, M. P., Grossmann, S. \& Lohse, D. 1998. Analysis of Rayleigh-Plesset dynamics for sonoluminescing bubbles. Journal of Fluid Mechanics, 365, 171-204.

Hodges, B., StreEt, R. \& ZANG, Y. A method for simulation of viscous, nonlinear, freesurface flows. In: 20th Symposium on Naval Hydrodynamics, 1996. 791-809.

HsiaO, C.-T. , 1996, "Numerical study of the Tip Vortex Flow over a Finite-Span Hydrofoil," Ph.D. Thesis, Department of Mechanical Engineering, The Pennsylvania State University.

Hsiao, C.-T. \& Chahine, G. 2008. Numerical study of cavitation inception due to vortex/vortex interaction in a ducted propulsor. Journal of Ship Research, 52, 114-123.

HsiaO, C.-T. \& CHAHINE, G. L. 2012. Effect of a propeller and gas diffusion on bubble nuclei distribution in a liquid. Journal of Hydrodynamics, Ser. B, 24, 809-822.

Hsiao, C.-T., Chahine, G. L. \& LiU, H.-L. 2003. Scaling effect on prediction of cavitation inception in a line vortex flow. Journal of fluids engineering, 125, 5360 .

HsiaO, C.-T., Choi, J.-K., Singh, S., Chahine, G., HaY, T., IlinskiI, Y. A., Zabolotskaya, E., Hamilton, M., Sankin, G. \& Yuan, F. 2013a. Modelling single-and tandem-bubble dynamics between two parallel plates for biomedical applications. Journal of Fluid Mechanics, 716, 137-170.

Hsiao, C.-T., LU, X. \& Chahine, G. 2010. Three-dimensional modeling of the dynamics of therapeutic ultrasound contrast agents. Ultrasound in medicine \& biology, 36, 2065-2079.

HsiaO, C.-T. \& PAUley, L. L. 1998. Numerical study of the steady-state tip vortex flow over a finite-span hydrofoil. Journal of fluids engineering, 120, 345-353.

Hsiao, C. \& Chahine, G. 2001. Numerical Simulation of Bubble Dynamics in a Vortex Flow Using Moving Chimera Grid and Navier-Stokes Computations. CAV2001, Pasadena, CA. 
Hsiao, C. T., Wu, X., Ma, J. \& Chahine, G. L. 2013b. Numerical and experimental study of bubble entrainment due to a horizontal plunging jet. International Shipbuilding Progress, 60, 435-469.

Jayaprakash, A., Singh, S. \& Chahine, G. 2011. Experimental and Numerical Investigation of Single Bubble Dynamics in a Two-Phase Bubbly Medium. Transactions of the ASME-I-Journal of Fluids Engineering, 133, 121305.

Johnson, J. V. E., Chahine, G. L., Lindenmuth, W. T., Conn, A. F., Frederick, G. S. \& Giacchino, J. G. J. 1982. Cavitating and Structered Jets for Mechanical Bits to Increase Drilling Rate. The ASME Publication, 82-Pet-13, 1-16.

JOHnson, V. \& HSIEH, T. The influence of the trajectories of gas nuclei on cavitation inception. In: Sixth Symposium on Naval Hydrodynamics, 1966. 163-179.

Kalumuck, K., Chahine, G., HsiaO, C. \& Choi, J. Remediation and disinfection of water using jet generated cavitation. In: 5th International Symposium on Cavitation (CAV 2003), Osaka, Japan, 2003.

Kalumuck, K. M. \& Chahine, G. L. 2000. The use of cavitating jets to oxidize organic compounds in water. Journal of Fluids Engineering-Transactions of the Asme, $122,465-470$.

Keller, J. B. \& KolodneR, I. I. 1956. Damping of underwater explosion bubble oscillations. Journal of Applied Physics, 27, 1152-1161.

KelleR, J. B. \& MiKsis, M. 1980. Bubble oscillations of large amplitude. The Journal of the Acoustical Society of America, 68, 628.

KENNEDY, J. E. 2005. High-intensity focused ultrasound in the treatment of solid tumours. Nat Rev Cancer, 5, 321-7.

Kitagawa, A., Murai, Y. \& Yamamoto, F. 2001. Two-way coupling of EulerianLagrangian model for dispersed multiphase flows using filtering functions. International journal of multiphase flow, 27, 2129-2153.

KRIEGER, J. R. \& CHAHINE, G. L. 2005. Acoustic signals of underwater explosions near surfaces. The Journal of the Acoustical Society of America, 118, 2961.

Lauer, E., Hu, X., Hickel, S. \& AdAMS, N. 2012. Numerical modelling and investigation of symmetric and asymmetric cavitation bubble dynamics. Computers \& Fluids, 69, 1-19.

LAw, D., Battaglia, F. \& Heindel, T. J. Stability issues for gas-liquid flows in bubble columns. In: ASME 2007 International Mechanical Engineering Congress and Exposition, 2007. American Society of Mechanical Engineers, 53-61.

Law, D., Battaglia, F. \& Heindel, T. J. 2008. Model validation for low and high superficial gas velocity bubble column flows. Chemical Engineering Science, 63, 4605-4616.

Law, D., Jones, S. T., Heindel, T. J. \& Battaglia, F. 2011. A Combined Numerical and Experimental Study of Hydrodynamics for an Air-Water External Loop Airlift Reactor. Journal of fluids engineering, 133, 021301.

Loraine, G., Chahine, G., Hsiao, C.-T., Choi, J.-K. \& Aley, P. 2012. Disinfection of gram-negative and gram-positive bacteria using DynaJets ${ }^{\circledR}$ hydrodynamic cavitating jets. Ultrasonics sonochemistry, 19, 710-717. 
Ma, J., Hsiao, C.-T. And Chahine, G.L., 2015. Euler-Lagrange Simulations of Bubble Cloud Dynamics near a Wall, ASME J. Fluids Eng., 137(4), pp. 041301-10

MADADI-KANDJANI, E. \& XIONG, Q. 2014. Validity of the spring-backed membrane model for bubble-wall interactions with compliant walls. Computers \& Fluids, 96, 116-121.

MiKsis, M. J. \& TING, L. 1984. Nonlinear radial oscillations of a gas bubble including thermal effects. The Journal of the Acoustical Society of America, 76, 897.

MitragotRI, S. 2005. Innovation - Healing sound: the use of ultrasound in drug delivery and other therapeutic applications. Nature Reviews Drug Discovery, 4, 255-260.

Moholkar, V., Senthil Kumar, P. \& Pandit, A. 1999. Hydrodynamic cavitation for sonochemical effects. Ultrasonics Sonochemistry, 6, 53-65.

MoRCH, K.A., 1981, Cavity cluster dynamics and cavitation erosion. Proc. ASME. Cavitation and Polyphase Flow Forum, pp. 1-10.

OH, J. M., KIM, P. \& KANG, I. S. 2001. Chaotic oscillation of a bubble in a weakly viscous dielectric fluid under electric fields. Physics of Fluids, 13, 2820.

Ohl, C. D., Arora, M., Dijkink, R., Janve, V. \& Lohse, D. 2006. Surface cleaning from laser-induced cavitation bubbles. Applied Physics Letters, 89.

Plesset, M. 1949. The dynamics of cavitation bubbles. J. appl. Mech, 16, 227-282.

Plesset, M. S. \& Prosperetti, A. 1977. Bubble dynamics and cavitation. Annual Review of Fluid Mechanics, 9, 145-185.

ProsperetTi, A. 1982. A generalization of the Rayleigh-Plesset equation of bubble dynamics. Physics of Fluids (1958-1988), 25, 409-410.

ProsperetTI, A. 1987. The equation of bubble dynamics in a compressible liquid. Physics of Fluids, 30, 3626.

ProsperetTI, A. 1991. The thermal behaviour of oscillating gas bubbles. Journal of Fluid Mechanics, 222, 587-616.

Prosperetti, A., Crum, L. A. \& Commander, K. W. 1988. Nonlinear bubble dynamics. The Journal of the Acoustical Society of America, 83, 502.

ProsperetTi, A. \& HAO, Y. 1999. Modelling of spherical gas bubble oscillations and sonoluminescence. Philosophical Transactions of the Royal Society of London. Series A: Mathematical, Physical and Engineering Sciences, 357, 203-223.

Prosperetti, A. \& LeZzI, A. 1986. Bubble dynamics in a compressible liquid. Part 1. First-order theory. J. FLUID MECH., 1986, 168, 457-478.

Raju, R., Singh, S., Hsiao, C.-T. \& Chahine, G. 2011. Study of Pressure Wave Propagation in a Two-Phase Bubbly Mixture. Transactions of the ASME-IJournal of Fluids Engineering, 133, 121302.

RAYLEIGH, L. 1917. VIII. On the pressure developed in a liquid during the collapse of a spherical cavity. The London, Edinburgh, and Dublin Philosophical Magazine and Journal of Science, 34, 94-98.

RoE, P. L. 1981. Approximate Riemann solvers, parameter vectors, and difference schemes. Journal of computational physics, 43, 357-372. 
Seo, J. H., Lele, S. K. \& Tryggvason, G. 2010. Investigation and modeling of bubblebubble interaction effect in homogeneous bubbly flows. Physics of Fluids, 22, 063302.

Shams, E., FInN, J. \& APTE, S. 2011. A numerical scheme for Euler-Lagrange simulation of bubbly flows in complex systems. International Journal for Numerical Methods in Fluids, 67, 1865-1898.

SingH, R. \& ShARMA, S. 2012. Development of suitable photobioreactor for algae production-A review. Renewable and Sustainable Energy Reviews, 16, 23472353.

Spelt, P. \& Biesheuvel, A. 1997. On the motion of gas bubbles in homogeneous isotropic turbulence. Journal of Fluid Mechanics, 336, 221-244.

Spelt, P. \& MAtar, O. 2006. Collapse of a bubble in an electric field. Physical Review E, 74, 046309.

SusSmAN, M. 2003. A second order coupled level set and volume-of-fluid method for computing growth and collapse of vapor bubbles. Journal of Computational Physics, 187, 110-136.

Van Leer, B., Thomas, J.L., Roe, P.L. And Newsome, R.W., 1987, “A Comparison of Numerical Flux Formulas for the Euler and Navier-Stokes Equation," AIAA Paper No. 87-1104-CP.

VAn Sint Annaland, M., DeEn, N. \& KuIPERS, J. 2005. Numerical simulation of gasliquid-solid flows using a combined front tracking and discrete particle method. Chemical Engineering Science, 60, 6188-6198.VAN WIJNGAARDEN, L., 1964. On the Collective Collapse of a Large Number of Gas Bubbles in Water", Proceedings 11th International Congress of Applied Mechanics, Springer, Berlin, pp.854-861.

VAN WiJnGAARDEN, L., 1968, On the Equations of Motion for mixtures of Liquid and Gas Bubbles, J. Fluid Mech. 33(3), pp.465-474

VAN WiJnGAARDEN, L., 1972, One-Dimensional Flow of Liquids Containing Small Gas Bubbles. Annual Review of Fluid Mechanics. 4, pp.369-396

WANG, Q. \& BlAKE, J. 2010. Non-spherical bubble dynamics in a compressible liquid. Part 1. Travelling acoustic wave. Journal of Fluid Mechanics, 659, 191-224.

WARDLAW, A. \& LUTON, J. Fluid-structure coupling methodology for undersea weapons. In: 2 nd International Conference on Fluid Structure Interaction, 2003. 251-261.

WARDLAW, J., ANDREW B \& LUTON, J. A. 2000. Fluid-structure interaction mechanisms for close-in explosions. Shock and Vibration, 7, 265-275.

Wardlaw, J., ANDrew B \& MaIR, H. U. 1998. Spherical solutions of an underwater explosion bubble. Shock and Vibration, 5, 89-102.

Wu, X., Choi, J.-K., Hsiao, C.-T. \& Chahine, G. L. Bubble augmented waterjet propulsion: Numerical and experimental studies. In: Proc. 28th Symposium on Naval Hydrodynamics, 2010.

Wu, X., Choi, J.-K., Singh, S., HsiaO, C-T, and Chahine, G. L. , 2012. Experimental and Numerical Investigation of Bubble Augmented Waterjet Propulsion. J. of Hydrodynamics, 24(5), pp.635-647. 
WU, Y. \& GIDASPOW, D. 2000. Hydrodynamic simulation of methanol synthesis in gasliquid slurry bubble column reactors. Chemical Engineering Science, 55, 573587.

Xiang, M., Cheung, S., Yeoh, G., Zhang, W. \& Tu, J. 2011. On the numerical study of bubbly flow created by ventilated cavity in vertical pipe. International Journal of Multiphase Flow, 37, 756-768.

XIANG, M., JiAng, Z., Zhang, W. \& Tu, J. 2014. Numerical Modeling and Analysis of Gas Entrainment for the Ventilated Cavity in Vertical Pipe. Chinese Journal of Chemical Engineering, 22, 252-260.

YASUI, K. 1999. Effect of a magnetic field on sonoluminescence. Physical Review E, 60, 1759.

YuAN, F., SANKIN, G. \& ZHONG, P. 2011. Dynamics of tandem bubble interaction in a microfluidic channel. The Journal of the Acoustical Society of America, 130, 3339-3346.

Zhang, S., DunCAN, J. H. \& Chahine, G. L. 1993. The final stage of the collapse of a cavitation bubble near a rigid wall. Journal of Fluid Mechanics, 257, 147-181.

Zhong, P., ZhOU, Y. \& ZHU, S. 2001. Dynamics of bubble oscillation in constrained media and mechanisms of vessel rupture in SWL. Ultrasound in medicine \& biology, 27, 119-134. 